\title{
Quantum trimer models and topological SU(3) spin liquids on the kagome lattice
}

\author{
Sven Jandura $\odot,,^{1,2,3}$ Mohsin Iqbal $\odot,{ }^{3,4}$ and Norbert Schuch ${ }^{3,4}$ \\ ${ }^{1}$ Ludwig-Maximilians-Universität, Schellingstraße 4, 80799 München, Germany \\ ${ }^{2}$ Institute for Theoretical Physics, ETH Zürich, Wolfgang-Pauli-Strasse 27, 8093 Zürich, Switzerland \\ ${ }^{3}$ Max-Planck-Institute for Quantum Optics, Hans-Kopfermann-Straße 1, 85748 Garching, Germany \\ ${ }^{4}$ Munich Center for Quantum Science and Technology, Schellingstraße 4, 80799 München, Germany
}

(Received 19 June 2020; revised 13 August 2020; accepted 14 August 2020; published 10 September 2020)

\begin{abstract}
We construct and study quantum trimer models and resonating SU(3)-singlet models on the kagome lattice, which generalize quantum dimer models and the resonating valence bond wave functions to a trimer and SU(3) setting. We demonstrate that these models carry a $\mathbb{Z}_{3}$ symmetry which originates in the structure of trimers and the SU(3) representation theory, and which becomes the only symmetry under renormalization. Based on this, we construct simple and exact parent Hamiltonians for the model which exhibit a topological ninefold degenerate ground space. A combination of analytical reasoning and numerical analysis reveals that the quantum order ultimately displayed by the model depends on the relative weight assigned to different types of trimers-it can display either $\mathbb{Z}_{3}$ topological order or form a symmetry-broken trimer crystal, and in addition possesses a point with an enhanced U(1) symmetry and critical behavior. Our results accordingly hold for the SU(3) model, where the two natural choices for trimer weights give rise to either a topological spin liquid or a system with symmetry-broken order, respectively. Our work thus demonstrates the suitability of resonating trimer and SU(3)-singlet ansatzes to model SU(3) topological spin liquids on the kagome lattice.
\end{abstract}

DOI: 10.1103/PhysRevResearch.2.033382

\section{INTRODUCTION}

Spin liquids are exotic phases of matter where the competition between strong antiferromagnetic interactions and geometric frustration prevents magnetic ordering, but instead gives rise to topological order, that is, a global ordering in the structure of their entanglement, and which thus display a range of exotic properties such as fractional excitations with exotic statistics [1-3]. A paradigmatic model for topological spin liquids has been Anderson's resonating valence bond (RVB) state [4]. It is constructed from different coverings of the lattice with $\mathrm{SU}(2)$ spin- $1 / 2$ singlets, which are placed in a "resonating" superposition such as to further lower their energy. Yet, the study of RVB wave functions poses the challenge that different singlet coverings are not orthogonal. To alleviate this problem, quantum dimer models have been studied instead, where inequivalent singlet configurations are taken to be orthogonal, such as in a large-spin limit [5].

The study of dimer models reveals a strong dependence of their quantum order on the type of lattice. Specifically, dimer models on bipartite lattices are generally critical [5,6], while conversely on nonbipartite lattices, in particular the triangular and kagome lattice, they have been found to exhibit $\mathbb{Z}_{2}$

Published by the American Physical Society under the terms of the Creative Commons Attribution 4.0 International license. Further distribution of this work must maintain attribution to the author(s) and the published article's title, journal citation, and DOI. Open access publication funded by the Max Planck Society. topological order $[7,8]$. Here, the case of the kagome lattice is of particular interest: First, the dimer model on the kagome lattice is a renormalization (RG) fixed point with a direct mapping to a $\mathbb{Z}_{2}$ loop model [9] (i.e., Kitaev's toric code [10]), and second, kagome Heisenberg antiferromagnets are approximately realized in actual materials such as herbertsmithite [3,11], making the RVB state on the kagome lattice, despite not being their exact ground state wave function, a particularly interesting model to study. By using Tensor network methods, which allow to construct smooth interpolations from the RVB to the dimer model and which provide a powerful toolbox to directly probe for topological order, the topological nature of the kagome RVB state could be unambiguously shown, and the underlying parent Hamiltonians of the model were identified $[12,13]$.

Both the presence of $\mathbb{Z}_{2}$ topological order and the critical physics found for bipartite lattices can be understood from the symmetries of the system. Dimer models naturally exhibit a $\mathbb{Z}_{2}$ gauge symmetry - the parity of the number of dimers leaving any given region is fixed, arising from the fact that each dimer inside the region uses up two sites, or that two spin- $1 / 2$ make up a singlet—suggestive of $\mathbb{Z}_{2}$ topological order. On the other hand, on bipartite lattices, the $\mathbb{Z}_{2}$ is enhanced to a U(1) symmetry - the number of $A$ and $B$ sublattice dimers leaving a region must be equal, since each (nearest-neighbor) dimer in the region uses up an $A$ and a $B$ site-indicative of critical behavior. Thus identifying the symmetries displayed by the dimer model on different lattices is key to its understanding.

In recent years, $\mathrm{SU}(N)$ spin systems have received increasing interest, in particular due to the possibility to engineer $\mathrm{SU}(N)$-invariant interactions in the fundamental 
representation in experiments with cold atomic gases [14-16]. Hence, a natural first step is to consider SU(3)-symmetric models in the fundamental representation. In that case, singlets are tripartite, namely the fully antisymmetric state $\sum_{i j k} \varepsilon_{i j k}|i, j, k\rangle$. Just as in the RVB construction, we can think of building resonating $\mathrm{SU}(3)$ singlet states - that is, superpositions of different ways to cover the lattice with singlets- to lower the energy, and just as for the RVB state, in the attempt to analyze the model we are faced with the challenge that different singlet configurations are lacking orthogonality. It is therefore natural-again in analogy to the RVB state-to study quantum trimer models, where different SU(3) singlets are replaced by orthogonal trimer configurations. In this context, a few questions naturally arise: First, what is the role of the underlying lattice-for instance, is there a similar dichotomy in the type of order displayed on bipartite versus nonbipartite lattices? Second, is there a "natural" lattice on which to define the trimer model? This could either be a lattice on which the model forms an RG fixed point with a direct mapping to a loop model, such as the kagome lattice for the RVB state, or a lattice where the tripartite SU(3) singlets appear in a particularly natural way, which suggests a lattice built from triangular simplices and thus yet again the kagome lattice.

Previous work on trimer models and resonating SU(3) singlet wave functions has been focused on the square lattice. In particular, in Ref. [17], a quantum trimer model on the square lattice has been introduced and it has been found that it is topologically ordered, and in Ref. [18], a corresponding model with resonating SU(3) singlets on the square lattice has been identified as a $\mathbb{Z}_{3}$ topological spin liquid; both of these works employed numerical analysis based on tensor networks. Indeed, such an order could have been expected, since trimers and SU(3) singlets display a natural $\mathbb{Z}_{3}$ symmetry, analogous to the $\mathbb{Z}_{2}$ symmetry in dimers. On the other hand, in Ref. [19], an SU(3) spin liquid on the kagome lattice had been proposed whose "orthogonal" version is an RG fixed point, namely the $\mathbb{Z}_{3}$ loop gas version of the toric code, and which was shown to be topological. While this model itself did not allow for an interpretation as a superposition of trimer patterns, a modification of the SU(3) model which gave rise to such an interpretation was also discussed, and numerically found to be in a trivial phase. Together, these findings raise a number of questions: What is the nature of an orthogonal trimer model on the kagome lattice, whose simplices naturally support trimers? Can we explain the different behavior seen for square versus kagome lattice from underlying symmetries in the model; in particular, are there additional symmetries on top of $\mathbb{Z}_{3}$ emerging on the kagome lattice which speak against topological order? And finally, can we obtain a more general understanding of the way in which lattice geometries and the type of order in resonating $\mathrm{SU}(N)$ wave functions could be related?

In this paper, we present a systematic analytical and numerical study of the trimer model and its SU(3) variants on the kagome lattice. Our key results are as follows. (1) We present a simple mapping from the trimer model to a $\mathbb{Z}_{3}$ loop model (an "arrow representation" similar to the kagome RVB [9]), which however is missing a vertex configuration. However, as we show analytically, all $\mathbb{Z}_{3}$-invariant loop configurations re-appear under blocking. This shows that the model has the right symmetry to exhibit $\mathbb{Z}_{3}$ topological order, and that no additional symmetries are present which would point to a different phase. This forms the starting point for our subsequent analysis.

(2) We demonstrate that both the trimer and the SU(3) model can exhibit either $\mathbb{Z}_{3}$ topological order or conventional symmetry-breaking order, as well as critical behavior. Which one is realized is determined by the relative weight $\zeta$ of different types of trimers (specifically, those on the elementary simplices versus the rest). There are two natural choices for this weight, motivated by different interpretations of an equal weight superposition. For the quantum trimer model, we find that it is topologically ordered for both of these choices. The resonating SU(3) singlet model, on the other hand, is topologically ordered for one choice of $\zeta$ but breaks lattice symmetries for the other (the latter was observed in Ref. [19]). We further show that setting $\zeta$ to zero gives rise to an additional U(1) symmetry which results in a critical model. Finally, we provide phase diagrams for both the trimer and the SU(3) model as a function of the weight $\zeta$, as well as a detailed analysis of the behavior as we interpolate between the trimer and the SU(3) model.

(3) We explicitly construct exact parent Hamiltonians for the trimer model. In the arrow (i.e., loop model) representation, those Hamiltonians consist of 3-body terms across vertices and either 6-body or 11-body terms which act around one or two hexagons, respectively. For the 11-body Hamiltonians, we prove that they give rise to the correct ninefold degenerate topological ground space. Restricting to 6-body terms (the same locality as for the RG fixed point loop model) gives rise to 6 additional "frozen" classical ground states where the trimers display crystalline order, and which do not couple to the remaining nine ground states; moreover, we show that introducing a single 11-body term anywhere in the lattice allows to melt those crystals and recover the original ninefold degenerate ground space. Decorating the trimers with SU(3) singlets increases the locality of these terms to 8 and 12 , respectively.

Together, our results show a remarkably rich behavior of the quantum trimer and resonating SU(3)-singlet models on the kagome lattice, and refute a simple connection between the type of order and the geometry of the lattice for trimer models analogous to the dimer case. Moreover, our construction allows us to obtain a simple SU(3) spin liquid ansatz on the kagome lattice which has a natural interpretation as a resonating singlet pattern and thus as a superposition of simple product states which are connected through local moves. Finally, it highlights the importance of a new aspect in trimer models, and more generally $N$-mer models, as opposed to dimer models, namely, the key role played by the relative weights assigned to different trimer configurations.

The paper is structured as follows. In Sec. II, we introduce quantum trimer models, their arrow representation, and their loop gas representation. In Sec. III, we analyze the symmetry of the trimer model in the loop picture and show that the full $\mathbb{Z}_{3}$ symmetry (technically, $\mathbb{Z}_{3}$-injectivity [20]) is restored under blocking, which proves the absence of additional symmetries and implies the existence of local parent Hamiltonians. We then proceed to construct the simplest such 

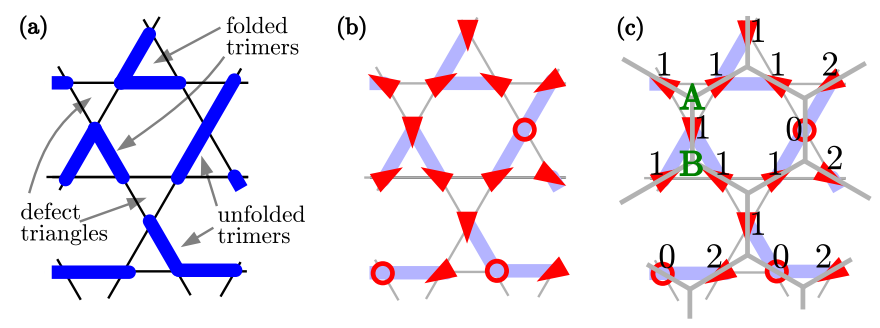

FIG. 1. (a) A trimer covering T. Folded trimers can be either treated as different depending on their orientation (as the two shown), or as identical (i.e., interpreted as a set of three vertices). We take the latter approach but allow to assign a different weight $\zeta$ to folded trimers, which allows to resolve them internally as a superposition of inequivalent trimers. (b) Arrow representation: We assign arrows to the outer vertices, pointing into the triangle towards the center of the trimer, o to inner vertices, and three inpointing arrows to folded trimers. This establishes a one-to-one mapping from trimer coverings to arrow patterns which obey a Gauss law, where the configuration with three $\circ$ is forbidden. (c) Loop representation. Arrows are replaced by 1 and 2, respectively, depending whether they point from the $A$ to the $B$ sublattice or vice versa, and $\circ$ by 0 . The loop representation again satisfies a Gauss law with 000 forbidden.

parent Hamiltonian, with the technical arguments given in two appendices. In Sec. IV, we combine analytical reasoning with numerical tensor network methods to investigate the type of order which the quantum trimer model exhibits, and in particular how the nature of the phase-topological, symmetry broken, or critical-depends on the relative weight of different trimers. Finally, in Sec. V, we generalize the trimer model by equipping it with $\mathrm{SU}(3)$ singlets, which we then continuously connect to a pure resonating SU(3)-singlet model with the fundamental representation acting on each site. We study the phase diagram of the SU(3) model as well as the physics along the trimer $\leftrightarrow \mathrm{SU}(3)$ interpolation, and discuss the corresponding parent Hamiltonian. The section closes with a brief discussion of the SU(3) model as a variational ansatz.

\section{TRIMER MODELS}

Let us start by defining trimer models, Fig. 1(a). A trimer on the kagome lattice is a covering of two adjacent edges and correspondingly three vertices. For a trimer, we distinguish two outer and one inner vertex, and we distinguish folded trimers (living on a single triangle) from unfolded ones (living on two triangles). A trimer covering $T$ is a complete covering of the lattice with nonoverlapping trimers, i.e., each vertex is contained in exactly one trimer. We can treat the trimer coverings $T$ as an orthonormal basis $|T\rangle$ of a Hilbert space; an (orthogonal) trimer model is then given as the equal-weight superposition of all trimer configurations $\mathcal{T}$,

$$
\left|\Psi_{\text {trimer }}\right\rangle=\sum_{T \in \mathcal{T}}|T\rangle .
$$

A key question will be how to treat folded trimers: Do we think of them as three different trimers (related by rotation), or do we treat them all as the same trimer (considering a trimer rather as an unordered set of three vertices), see Fig. 1(a)?
Here, we will treat folded trimers as equivalent (i.e., as a set of vertices), but we will allow to assign them a different weight $\zeta$ [cf. Eq. (2)], accounting for multiple "internal" degrees of freedom such as differently oriented trimers (e.g., $\zeta=\sqrt{3}$ would allow to resolve folded trimers in three orthogonal ways with weight 1 each).

A local representation of $|T\rangle$ can be constructed by assigning either arrows $\{\boldsymbol{\bullet}\}$ or a no-arrow symbol $\circ$ to every vertex [see Fig. 1(b)]: to each outer vertex of a trimer, we assign an arrow pointing into the triangle to which the trimer extends, and to inner vertices, we assign $\circ$. The exception are folded trimers, in which case we assign inpointing arrows to all three vertices. This associates a unique arrow pattern to each trimer covering. Conversely, any arrow pattern for which (i) the number of inpointing minus outpointing arrows is 0 mod 3 and (ii) triangles with three $\circ$ are forbidden, maps uniquely to a trimer pattern.

Let us now consider the arrow degrees of freedom as link variables on the dual honeycomb lattice. We replace the arrows by 1 and $2 \equiv-1$ (we work $\bmod 3$ from now on), depending on whether they point from the $A>B$ sublattice of the honeycomb or vice versa; $\circ$ will be replaced by 0 . The arrows then correspond to configurations with a $\mathbb{Z}_{3}$ Gauss law around each vertex of the honeycomb lattice, where the configuration 000 is forbidden. In this language, the trimer model corresponds to

$$
\left|\Psi_{\text {trimer }}\right\rangle=\sum_{L \in \mathcal{L}} \zeta^{N_{222}^{A}+N_{111}^{B}}|L\rangle,
$$

where the $\mathbb{Z}_{3}$ edge configurations $L$ on the honeycomb lattice are taken from the set $\mathcal{L}$ of all Gauss law configurations ("loop configurations") with no 000 around any vertex; here, $\zeta$ controls the relative weight of folded trimers (which correspond to 222 configurations around $A$ vertices and 111 around $B$ vertices, counted by $N_{222}^{A}$ and $N_{111}^{B}$ ).

To study the properties of this model it is convenient to use a tensor network or PEPS (projected entangled pair state) representation, which provides us with a range of powerful analytical and numerical tools for its analysis [20-26]. The corresponding PEPS is constructed from two types of tensors, see Fig. 2(a), similar to, e.g., the tensor network for the RVB and dimer model [12]: one type of tensor-corresponding to a state $|\tau\rangle=\sum t_{i j k}|i, j, k\rangle$-only has virtual indices and sits inside triangles, it ensures only valid vertex configurations appear (with weight $\zeta$ assigned to on-site triangles), that is, $t_{i j k}=\left|\varepsilon_{i j k}\right|+\delta_{i=j=k=1}+\zeta \delta_{i=j=k=2}$. The other tensorcorresponding to a map $\mathcal{P}=\sum P_{i j}^{a}|a\rangle\langle i, j|$-sits on the edges and maps two virtual degrees of freedom, one from each of the adjacent tensors, to a physical arrow state $|a\rangle$, that is, $P_{12}^{\nabla}=$ $P_{21}^{\boldsymbol{\Delta}}=P_{00}^{\circ}=1$ and zero otherwise, with the arrow oriented towards the 2 . The trimer wave function $\left|\Psi_{\text {trimer }}\right\rangle$ is then obtained by arranging the tensors on the honeycomb lattice and contracting the virtual indices, or alternatively applying the maps $\mathcal{P}$ to the states $|\tau\rangle$, see Fig. 2(a). [Note that on $B$ triangles, the role of 1 and 2 is swapped relative to Fig. 1(c); this can be changed by an appropriate gauge transformation.) 
(a)

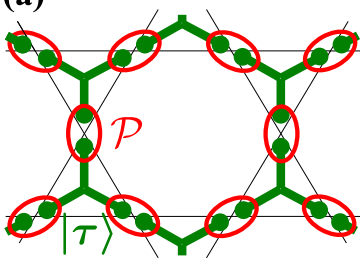

(b)

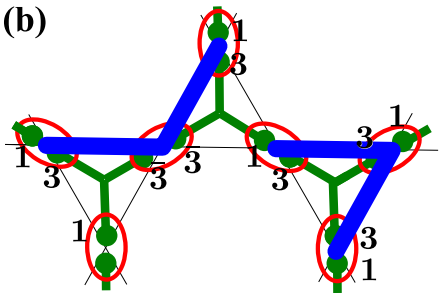

FIG. 2. (a) PEPS construction for the trimer and SU(3) model: the wave function is constructed by starting with fiducial states $|\tau\rangle$, which enforce the Gauss law, and subsequently applying maps $\mathcal{P}$ to them which output the physical degrees of freedom on the kagome lattice. (b) Construction of a trimer model with SU(3) degrees of freedom: the fiducial states transform as a singlet in $(\mathbf{1} \oplus \mathbf{3} \oplus \overline{\mathbf{3}})^{\otimes 3}$, where arrows point from $\mathbf{1}$ to $\mathbf{3}$ and thus the singlet in $\overline{\mathbf{3}} \otimes \overline{\mathbf{3}} \otimes \overline{\mathbf{3}}$ is forbidden; it is thus either a $\mathbf{3} \otimes \mathbf{3} \otimes \mathbf{3}$ singlet or a singlet in one of the pairs $\mathbf{3} \otimes \overline{\mathbf{3}} \otimes \mathbf{1}$. The resulting state is a trimer model decorated with SU(3) degrees of freedom. By projecting onto the $\mathbf{3}$ in $\overline{\mathbf{3}} \otimes \overline{\mathbf{3}}=$ $\mathbf{3} \oplus \overline{\mathbf{6}}$, trimers are decorates with $\mathrm{SU}(3)$ singlets.

\section{III. $\mathbb{Z}_{3}$-INJECTIVITY AND PARENT HAMILTONIAN}

We will now address the question whether the model is topologically ordered, and whether it appears as a ground state of a local parent Hamiltonian with a suitable (topological) ground space structure. To start with, the modified model which is an equal weight superposition of all Gauss law patterns-where we include the 000 configuration and let $\zeta=1$-is a topological RG fixed point model, namely, the $\mathbb{Z}_{3}$ version of Kitaev's toric code model [10]. But how severe is the exclusion of the 000 configuration? Does it induce a stronger symmetry than the $\mathbb{Z}_{3}$ Gauss law-e.g., a $\mathrm{U}(1)$ conservation law which would be indicative of a critical phase-or does it merely induce a finite length scale without breaking the topological order?

To understand this, we study what happens as we block sites: Does the missing 000 configuration result in missing configurations at all length scales - which would suggest additional conservation laws-or is the full symmetry restored? To this end, we consider a dual "height" representation of the model, where we assign $\mathbb{Z}_{3}$ variables to the plaquettes [Fig. 3(a), where the plaquette variables are labeled by roman letters], such that the edge degrees of freedom are obtained as the difference of plaquette variables (oriented clockwise/counter-clockwise around $B / A$ sublattice sites). This mapping from plaquettes to edges is 3-to-1, where the forbidden 000 configuration rules out three identical plaquette variables around a vertex [Fig. 3(b)]. Now consider first the neighborhood of one hexagon shown in Fig. 3(c). Given labels $a, \ldots, f$ at the boundary, when can we assign an allowed label to the central plaquette? It is easy to see that this is the case if and only if the pattern is not of the form Fig. 3(d) for $a, b, c$ all different, or rotations thereof. Now consider the block in Fig. 3(e), with arbitrary plaquette variables $\left\{a_{i}\right\}$ and $\left\{f_{j}\right\}$ assigned to the boundary-corresponding to an arbitrary boundary configuration of the edges which satisfies the $\mathbb{Z}_{3}$ Gauss law. Next, for $f_{i}=0,1,2$ assign $g_{i}=1,2,1$. This way, $f_{i} \neq g_{i}$ [and thus the condition Fig. 3(b) is satisfied around all vertices marked $\star]$, and $g_{i} \neq 0$. Finally, we assign 0 to the central plaquette. We now immediately see that we cannot

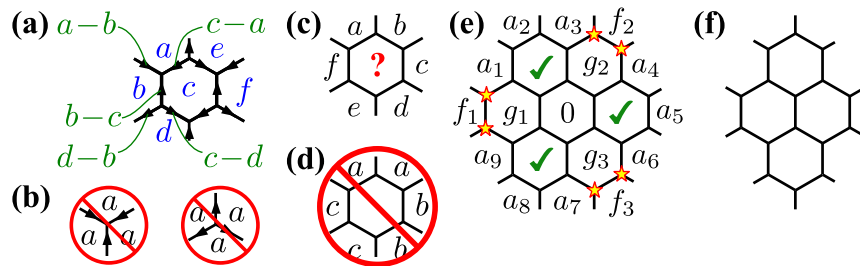

FIG. 3. (a) Height representation of the trimer model: a $\mathbb{Z}_{3}$ "height variable" (blue labels) is associated to every plaquette such that link variables (green labels) are the (oriented) differences of the height variables. (b) Forbidden height configurations (corresponding to the forbidden 000 configurations of the edges). [(c) and (d)] The central plaquette in (c) can be assigned a valid value exactly if the boundary is not in the state (d) or rotations thereof, with $a, b, c$ all different. (e) Region used for the $\mathbb{Z}_{3}$-injectivity proof (see text). (f) Smallest $\mathbb{Z}_{3}$-injective region, i.e., where all boundary configurations are admissible.

have the pattern of Fig. 3(d) around the plaquettes marked with green tickmarks, and thus, we can also assign consistent value to these. That is, any $\mathbb{Z}_{3}$-invariant loop configuration at the boundary has a realization on the interior, and is thus an admissible boundary configuration: We thus find that the $\mathbb{Z}_{3}$-invariant space is restored after blocking, proving that removing the 000 vertices from the $\mathbb{Z}_{3}$ loop model induces no additional constraints under renormalization. In fact, this result still holds for the smaller patch in Fig. 3(f), as can be verified by an exhaustive search.

The fact that the $\mathbb{Z}_{3}$-invariant subspace is restored under blocking - or, in the language of tensor networks, $\mathbb{Z}_{3}-$ injectivity [20] is reached (that is, the blocked tensor describes an injective map from boundary to bulk on the $\mathbb{Z}_{3}$-invariant subspace) implies the existence of a local parent Hamiltonian with a ninefold degenerate ground space on the torus, which is spanned by the trimer state $\left|\Psi_{\text {trimer }}\right\rangle$ and its topologically equivalent siblings, obtained by assigning a phase $\omega^{\nu_{h} N_{h}+v_{v} N_{v}}$ $\left(\omega=e^{2 \pi i / 3}\right)$ with $v_{h}, v_{v}=0,1,2$ to configurations for which the link variables sum to $N_{h}\left(N_{v}\right)$ along a horizontal (vertical) loop around the torus [12,20]. Note, however, that this does not necessarily imply that the model is topologically ordered in the thermodynamic limit (ground states can vanish, or additional low energy state can appear) [27], and numerical study is required in addition to unambigously assess the topological nature of the trimer model; we will turn to this in the next section.

For now, let us discuss the form of the parent Hamiltonian. Generally, the local terms in the parent Hamiltonian are positive semi-definite operators (e.g., projectors) which are constructed such that they are zero exactly on all allowed states on the spins supporting them (in a tensor network, this is the space spanned by choosing arbitrary boundary conditions) [20,24]. For the case of looplike models like the one at hand, one way to explicitly construct such Hamiltonians is to build them from two types of terms: The first consists of three-body projectors which act across vertices and have precisely the allowed vertex configurations in their kernel; here, those are the $\mathbb{Z}_{3}$ Gauss law configurations except 000. The second type of terms couples different loop configurations through local transitions (that is, its ground space is spanned by the 
superposition $\left|\chi_{k}\right\rangle$ of coupled loop configurations with the correct relative weights, $\left.h=\mathbb{1}-\sum\left|\chi_{k}\right\rangle\left\langle\chi_{k}\right|\right)$, in such a way that any two loop configurations in the same topological sector (distinguished by their winding number, i.e., dual to the basis above) can be coupled through a sequence of such local moves induced by the individual Hamiltonian terms.

On what region do the latter type of Hamiltonian terms have to act? Using the $\mathbb{Z}_{3}$-injectivity after blocking, such regions can be constructed using canonical techniques; in essence, they need to contain an injective region plus a thin surrounding in a way which allows patching regions together $[12,20,24,28]$. Given the minimum injective region identified in Fig. 3(f), this gives terms acting on finite but still rather large regions. However, as we show in Appendix A, one can do much better. In order for the Hamiltonian to couple all configurations in a topological sector, it is sufficient to have Hamiltonian terms which act on two adjacent hexagons, or 11 edge degrees of freedom in the arrow or loop representation!

We thus find that in order to ensure a topologically degenerate ground space, one needs a Hamiltonian of the form

$$
H=\sum h_{\prec}+\sum h_{\infty},
$$

where $h_{\prec}$ is a projector acting on vertices which has the allowed vertex configurations as their ground space, and $h_{\infty}$ is a projector acting on pairs of adjacent plaquettes which, for every choice of surrounding degrees of freedom, has the equal weight superpositions of all allowed configurations consistent with those boundaries as their ground space; ${ }^{1}$ the sums run over all vertices and pairs of adjacent hexagons, respectively.

What happens when we go even further and restrict to one-hexagon Hamiltonians, $H=\sum h_{\prec}+\sum h_{\bigcirc}$, where $h_{\diamond}$ induces transitions between all allowed configurations on a hexagon (which is the same locality as for the $\mathbb{Z}_{3}$ fixed point loop model)? It turns out that in that case, additional ground states appear. Those states are all of the form shown in Fig. 4(a), that is, they exhibit crystalline order. ${ }^{2}$ Using the height representation (see figure), it can be easily seen that it is impossible to change the value of a single plaquette without violating Fig. 3(b), and an update acting on two hexagons is needed to start melting the crystal [shown red in Fig. 4(a)].

As it turns out-discussed in detail in Appendix B-these are the only additional ground states which appear when restricting to one-hexagon terms, while all other configurations in each topological sector can be coupled even by onehexagon updates. Differently speaking, crystals of the form Fig. 4(a), which only cover part of the system can be melted from the outside, and in particular, it is possible to change the configuration anywhere inside the crystal by melting a channel through the crystal, updating the configuration as needed, and re-freezing it towards the outside. This also implies that a single two-hexagon term $h_{\infty}$ anywhere in the system is sufficient to couple the crystalline configurations to

\footnotetext{
${ }^{1}$ Note that the latter term needs no access to the surrounding degrees of freedom: their value is unchanged and can be inferred from the degrees of freedom in the two hexagons using Gauss' law.

${ }^{2}$ There are six such states related by symmetry.
}

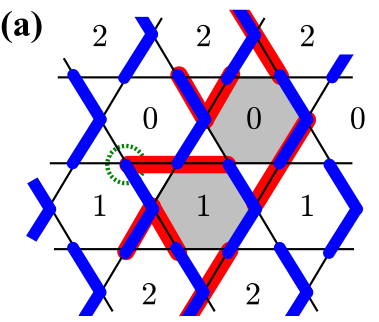

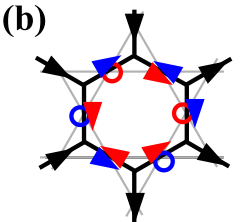

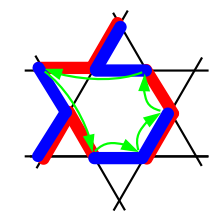

(c)

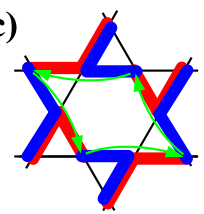

FIG. 4. (a) "Frozen" trimer configuration (blue) with crystalline order which cannot be melted by one-hexagon moves alone, and thus forms an independent ground state under one-hexagon parent Hamiltonians; this can be seen immediately from the height representation (labels inside hexagons). A two-hexagon move is needed to start melting the crystal, illustrated by the red trimer configuration which requires updating the two hexagons marked gray. (b) When equipping the trimers with $\mathrm{SU}(3)$ singlets, a Hamiltonian which acts on one hexagon in the arrow representation (left) also might have to act on some outer vertices due to the entanglement of the trimer (right). Here, the Hamiltonians acts by coupling the blue and red configurations. (c) For single-hexagon moves, in the worst case, it is required to act on two degrees of freedom outside of the hexagon. Similarly, for the two-hexagon move in (a), one has to act on at least one additional outside degree of freedom, as the one marked by the dashed green circle.

the others, and this way get back a Hamiltonian with nine ground states. ${ }^{3}$

\section{ORDER AND PHASE DIAGRAM}

Is the trimer model on the kagome lattice topologically ordered, and does this depend on the weight $\zeta$ of folded trimers? To this end, let us first see what we can understand analytically, e.g., in limiting cases, about the effect of varying $\zeta$. To start with, observe that any unfolded trimer uses up two triangles per three vertices. Folded trimers, on the other hand, only require one triangle per three vertices. As the kagome lattice has two triangles per three vertices, each folded trimer is therefore accompanied by exactly one defect triangle, that is, a triangle with no edge of a trimer on it [Fig. 1(a)]. Thus we find that the trimer state (2) is a sum over all trimer configurations, where configurations with each $K$ folded trimers and $K$ defect triangles are weighted by $\zeta^{K}$. (Curiously, this implies that only the product of the reweighting of folded trimers and defect triangles matters). From this, we can understand two limiting cases. For $\zeta \rightarrow \infty$, the system consists solely of folded trimers and defect triangles, and will thus break the lattice symmetry by putting all (folded) trimers either only on up- or only on down-pointing triangles; such a type of crystalline order has indeed been identified for the ground

\footnotetext{
${ }^{3}$ Note, however, that in this case configurations which only differ in one location are typically connected by a path of updates with a length on the order of the system size $N$, that is, in $N$ th order, which suggests that the corresponding system will be gapless.
} 
state of the SU(3) Heisenberg model on the kagome lattice (see also Sec. V). On the other hand, for $\zeta=0$, folded trimers and defect triangles disappear, and thus the configurations 111 and 222 are forbidden (in addition to the already forbidden 000). The remaining configurations are 012 and its permutations, i.e., one in- and one outpointing arrow at every vertex. At $\zeta=0$, the system thus possesses a U(1) symmetry, suggestive of critial behavior [as one can construct a $\mathrm{U}(1)$ height representation and thus an effective $\mathrm{U}(1)$ field theory description].

In order to understand the behavior of the system also away from those limiting cases, we use tensor network techniques introduced in earlier work to study the nature of the trimer model [19,26,29-31]. Specifically, we compute iMPS fixed points of the transfer matrix from left and right, and use their symmetry breaking pattern with respect to the $\mathbb{Z}_{3} \times \mathbb{Z}_{3}$ symmetry in ket+bra to identify the topological nature of the system; this method also allows us to extract correlation lengths $\xi$ for the trivial and anyon-anyon correlators (where the latter correspond to the inverse anyon mass).

First, we study an interpolation from the RG fixed point to the trimer model at $\zeta=1$, obtained by decreasing the weight of 000 configurations. This corresponds to a smooth interpolation of parent Hamiltonians (as $\mathbb{Z}_{3}$-injectivity is kept) [12], and is thus a reliable way to certify the absence of phase transitions, in addition to the symmetry breaking pattern of the topological $\mathbb{Z}_{3} \times \mathbb{Z}_{3}$ symmetry in the entanglement. The result is shown in Fig. 5(a), where the $x$ axis gives the weight of the 000 configuration in the superposition. We see that as we decrease the weight of the 000 configuration, correlations in the system build up, up to $\xi \approx 0.72$ for the trimer point. The dominant length scale is given by visons, while spinons or combined vison-spinon ("dyon") correlations remain zero. This is to be expected, as visons correspond to a disbalance in different Gauss law (i.e., loop) configurations (induced by suppressing 000), while spinons correspond to breaking up trimers (violations of the Gauss law), which doesn't occur as different trimers remain orthogonal. ${ }^{4}$

Let us next consider the phase diagram as a function of the weight $\zeta$ of folded trimers. Our results are shown in Fig. 6, where we plot trivial and (in the topological phase) vison correlations vs. $\zeta$. Together with the analysis of the ordering relative to the $\mathbb{Z}_{3}$ symmetry, we indentify a $\mathbb{Z}_{3}$ topological phase around $\zeta=1$, where we had already established topological order by interpolation from the RG fixed point. Around $\zeta_{c} \approx 2.19$, we observe a transition into a $\mathbb{Z}_{2}$ symmetry breaking phase- this is exactly the phase we discussed above, where for $\zeta \rightarrow \infty$, folded trimers and defect triangles alternate. On the other hand, for $\zeta \rightarrow 0$, the correlations diverge, consistent with critical behavior induced by the $\mathrm{U}(1)$ symmetry at $\zeta=0$ discussed above. Noteworthily, $\zeta_{c}>\sqrt{3}$, i.e., the trimer model is topologically ordered independent of whether we interpret folded trimers as a single object or as a sum of three orthogonal trimer realizations. The inset

${ }^{4}$ In the PEPS picture, vison pairs correspond to strings of $\mathbb{Z}_{3}$ symmetry actions, while spinons correspond to objects which transform as a nontrivial irrep under the symmetry, placed on the links when contracting tensors $[12,19,30,31]$.

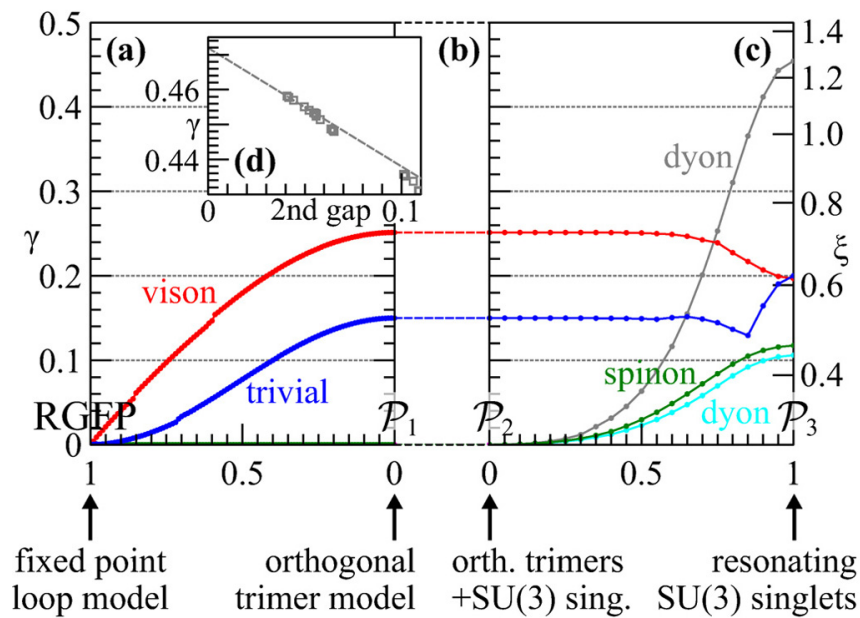

FIG. 5. Length scales $\xi$ for two-point correlations (labeled "trivial") and anyon-anyon correlators (i.e., inverse anyon masses, labeled by anyon type), along a sequence of interpolations. The $y$ axis is $\gamma=$ $e^{-1 / \xi}$. (a) Interpolation from the loop model ( $\mathrm{RG}$ fixed point) to the trimer model, by removing the 000 configuration. No spinon correlations appear, as different trimers configurations remain orthogonal. [(b) and (c)] Trimers are equipped with an SU(3) representation, see text: interpolation (b) removes the $\overline{\mathbf{6}}$ in the center of the trimer and has no effect, and yields trimers equipped with $\mathrm{SU}(3)$ singlets at the point $\mathcal{P}_{2}$. Interpolation (c) removes the arrow information; this gives rise also to spinon and dyon correlations as orthogonality of trimer configurations is lost. However, correlations remain finite all the way to the $\mathrm{SU}(3)$ point $\mathcal{P}_{3}$. (d) (Inset) Extrapolation of the leading correlation (dyon mass) at the SU(3) point for increasing iMPS bond dimension, using the $\varepsilon-\delta$ method [32].

shows the scaling behavior of the correlation length $\xi$ for a critical point $\zeta_{c}=2.188$, which yields a critical exponent $v \approx 2 / 3$. Remarkably, this is consistent with the four-state Potts transition.

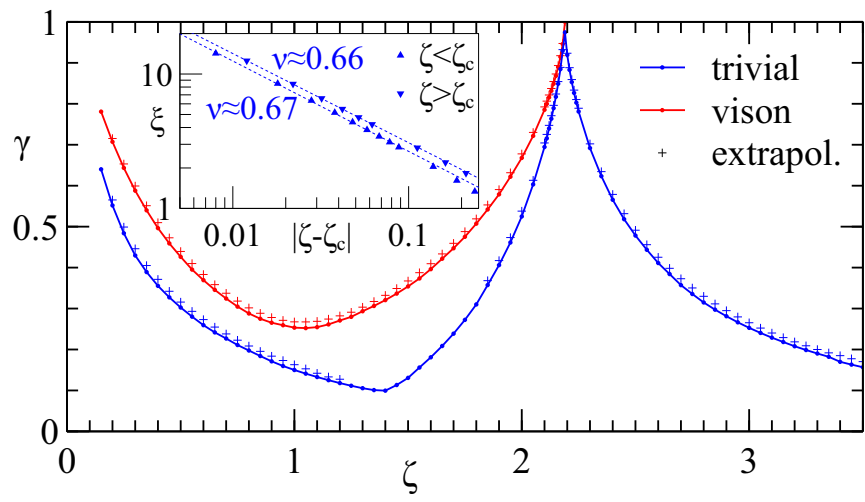

FIG. 6. Correlation length $\xi$ vs. folded trimer weight $\zeta$ for the trimer model. We plot $\gamma=e^{-1 / \xi}$ for trivial and vison correlations. We identify a $\mathbb{Z}_{3}$ topological phase for $\zeta<\zeta_{c} \approx 2.19$, a $\mathbb{Z}_{2}$ symmetry breaking phase for $\zeta>\zeta_{c}$, and a critical point at/around $\zeta=0$. Data shown is for an iMPS truncation error of $\eta=10^{-4} \times$ $2^{-38} \approx 3.64 \times 10^{-16}$, crosses give extrapolations obtained with the $\varepsilon-\delta$ method [32] (where reliable). The inset shows the critical scaling around $\zeta_{c}=2.188$ for the extrapolated values, which yields a critical exponent $v \approx 2 / 3$ at both sides of the transition. 


\section{SU(3) MODEL}

It is natural to use the trimer model to build an SU(3) model. If we attach the fundamental $\mathbf{3}$ representation to each vertex, an $\mathrm{SU}(3)$ singlet consists of three spins $\mathbf{3} \otimes \mathbf{3} \otimes \mathbf{3}$ and is of the form $\sum \varepsilon_{i j k}|i, j, k\rangle$ (with $\varepsilon$ the fully antisymmetric tensor). By replacing each trimer with an SU(3) singlet (suitably oriented), we thus arrive at an SU(3) resonating trimer state, in analogy to $\mathrm{SU}(2)$ resonating valence bond states.

Is the physics of the model affected by replacing the trimers by SU(3) singlets? Since unlike abstract trimers, different singlet configurations are not orthogonal, this is far from obvious. In order to assess this question, we employ a tensor network representation of the model which allows to treat the (orthogonal) trimer model and the SU(3) model on the same footing; it can be seen as a variant of the SU(3) model in Ref. [19], see Fig. 2(a). We start by triangular states $|\tau\rangle$ of three sites with representation $\mathbf{1} \oplus \mathbf{3} \oplus \overline{\mathbf{3}}$ each, where $|\tau\rangle$ is an equal weight superposition of the eight possible singlets without the one in $\overline{\mathbf{3}} \otimes \overline{\mathbf{3}} \otimes \overline{\mathbf{3}}$, and where we choose the amplitudes of all singlets +1 , except for the one in $\mathbf{3} \otimes \mathbf{3} \otimes \mathbf{3}$, which has amplitude $i \zeta$; this way, $|\tau\rangle$ is rotational invariant and transforms under reflection $\mathcal{R}$ as $\mathcal{R}|\tau\rangle=|\bar{\tau}\rangle$. Next, we place $|\tau\rangle$ on the simplices of the kagome lattice and apply maps $\mathcal{P}(\vec{\theta})$ which depend on some interpolation parameters $\vec{\theta}$. There are three special points for $\mathcal{P}$ between which we interpolate: $\mathcal{P}_{1}$ projects each site onto $(\mathbf{1} \otimes \mathbf{3}) \oplus(\mathbf{3} \otimes \mathbf{1}) \oplus$ $(\overline{\mathbf{3}} \otimes \overline{\mathbf{3}})$-this is unitarily equivalent to the (orthogonal) trimer model, by associating $\mathbf{1} \otimes \mathbf{3}$ to $\boldsymbol{\nabla}$, and $\overline{\mathbf{3}} \otimes \overline{\mathbf{3}}$ to $\circ$. (Gauss law is ensured since $|\tau\rangle$ is a singlet.) This assignment produces a direct correspondence between configurations of $|\tau\rangle$ and trimer patterns, cf. Fig. 2(b). Next, $\mathcal{P}_{2}$ is obtained from $\mathcal{P}_{1}$ by removing the $\overline{\mathbf{6}}$ from the subspace $\overline{\mathbf{3}} \otimes \overline{\mathbf{3}}=\mathbf{3} \oplus \overline{\mathbf{6}}$ while keeping the total weight of the subspace (i.e., reweighting it by $\sqrt{3}-$ not doing so would suppress $\overline{\mathbf{3}} \otimes \overline{\mathbf{3}}$ and thus unfolded trimers). After removing the $\overline{\mathbf{6}}$, the effective Hilbert space is $\mathbf{3} \oplus \mathbf{3} \oplus \mathbf{3} \cong \mathbf{3} \otimes \mathbb{C}^{3}$. Finally, $\mathcal{P}_{3}$ removes the degeneracy space $\mathbb{C}^{3}$ by projecting on the equal weight superposition of the three $\mathbf{3}$ (where the relative phases \pm 1 are chosen such that $\mathcal{P}_{3} \mathcal{R}=-\mathcal{P}_{3}$, which yields a rotationally invariant wave function $|\Psi\rangle$ which transform as $\mathcal{R}|\Psi\rangle=|\bar{\Psi}\rangle$ ). The interpolation $\mathcal{P}_{1} \rightarrow \mathcal{P}_{2} \rightarrow \mathcal{P}_{3}$ can be carried out continuously-where along the entire $\mathcal{P}_{1} \rightarrow \mathcal{P}_{2}$ interpolation, we keep the weight of the $\overline{\mathbf{3}} \otimes \overline{\mathbf{3}}$ subspace constant-allowing us to go smoothly from the orthogonal trimer model to the SU(3) model. ${ }^{5}$ We refer the reader to Ref. [19] for further details.

The behavior along the interpolation is shown in Figs. 5(b) and $5(\mathrm{c})$. Notably, interpolating $\mathcal{P}_{1} \rightarrow \mathcal{P}_{2}$ does not induce any change in the wave function due to the reweighting. This can be understood since $\overline{\mathbf{3}} \otimes \overline{\mathbf{3}}$ only appears in the middle of unfolded trimers, and different trimers remain orthogonal-the interpolation is thus merely a local basis transformation, while without the reweighting, unfolded trimers would have been suppressed. ${ }^{6}$ When interpolating $\mathcal{P}_{2} \rightarrow \mathcal{P}_{3}$, orthogonality of

\footnotetext{
${ }^{5}$ In Fig. 5, we plot the data vs. the amplitude which we change along the interpolation.

${ }^{6}$ Along the interpolation $\mathcal{P}_{1} \rightarrow \mathcal{P}_{2}$, the entangled state put on top of the trimer is - up to normalization-of the form $\left(11 \otimes W_{\theta} \otimes\right.$
}

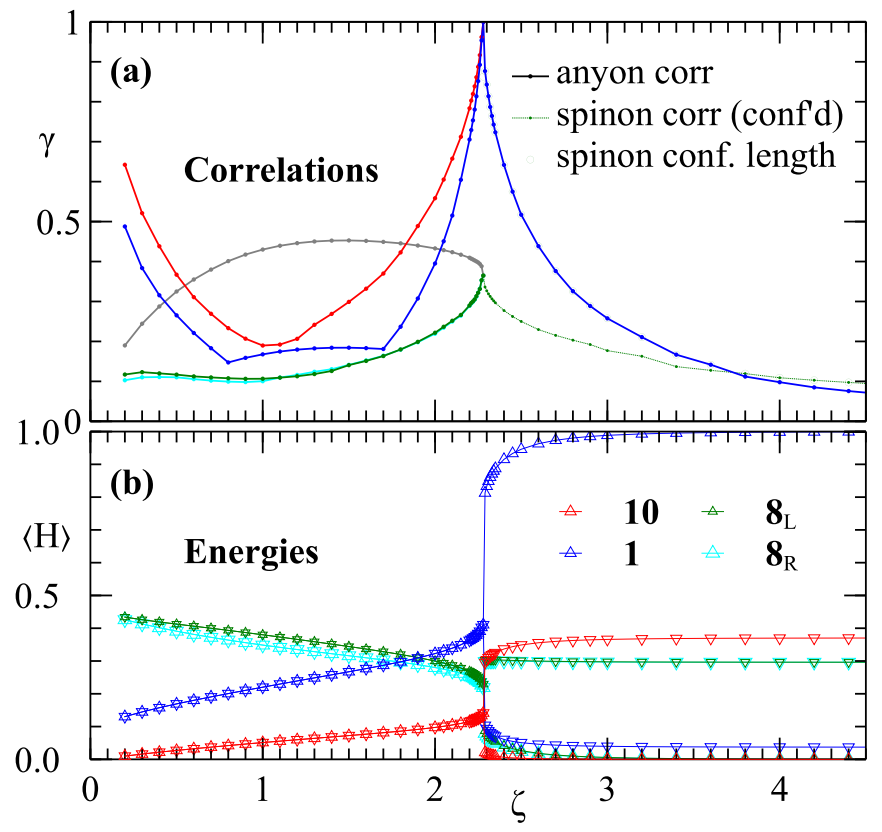

FIG. 7. (a) Correlation lengths for trivial and anyonic correlations vs. the folded trimer weight $\zeta$ for the SU(3) model, where trimers have been replaced by SU(3) singlets (i.e., fully antisymmetric states), cf. also Fig. 6. The color coding used is the same as in Fig. 5. We again identify a topological phase in the center, which transitions to a critical phase for $\zeta \rightarrow 0$, and to a symmetry-broken phase around $\zeta_{c} \approx 2.28$. In the symmetry broken phase on the right, spinons become confined, and the associated spinon confinement length is shown by open (green) circles. It first equals the twopoint (trivial) correlation length, and for large $\zeta \gtrsim 3.7$, the (now unphysical) correlation length between spinons. Data have been obtained with iMPS truncation threshold $\eta=10^{-4} \times 2^{-14} \approx 6.10 \times$ $10^{-9}$. (b) Variational energies vs $\zeta$ for the different SU(3) invariant terms on up $(\triangle)$ and down $(\nabla)$ triangles, see text.

different trimer configurations is lost, which induces a finite length scale also for spinons and combined dyonic excitations; we find that for the $\mathrm{SU}(3)$ point, the dominant length scale is a dyonic one, with $\xi \approx 1.3$. Importantly, from Fig. 5, together with an extrapolation of the correlations, it is clear that the correlations remain finite, and the $\mathrm{SU}(3)$ resonating trimer model is in the $\mathbb{Z}_{3}$ topological phase; for the $S U(3)$ point, the extrapolation [Fig. 5(d)] yields a dominant dyon correlation length of $\xi=1.33$.

Second, we have studied the dependence of the phase diagram on the folded trimer weight $\zeta$ at the $\mathrm{SU}(3)$ point $\mathcal{P}_{3}$. The results for the correlation lengths are shown in Fig. 7(a) (we again plot $\gamma=e^{-1 / \xi}$, with the same color coding for the different anyon sectors as in Fig. 5): We again identify a topological phase for $\zeta<\zeta_{c} \approx 2.28$, a symmetry broken valence bond crystal phase for $\zeta>\zeta_{c}$, and a critical phase at or around $\zeta=0$, which can be understood using the same qualitative picture as before for the trimer model. We find that

11) $(|\omega\rangle \otimes|\bar{\omega}\rangle)$, where $|\omega\rangle$ is the singlet in $\mathbf{3} \otimes \overline{\mathbf{3}},|\bar{\omega}\rangle$ the reflected version of $|\omega\rangle$, and $W_{\theta}$ decreases the weight of $\overline{\mathbf{6}}$ in $\overline{\mathbf{3}} \otimes \overline{\mathbf{3}}=\mathbf{3} \oplus \overline{\mathbf{6}}$ all the way to zero (which gives the antisymmetric state). 
the phase transitions out of the topological phase are driven by diverging vison correlations (i.e., vison condensation), just as for the trimer model, even though in the center of the topological phase around $\zeta=1$, dyonic correlations (which include a spinon contribution) are dominating. At the phase transition, the visons condense, leading to the confinement of spinons in the symmetry broken crystalline phase. Figure 7(a) also shows the associated spinon confinement length; interestingly, this length scale is initially dominated by the equally divergent trivial correlations, while around $\zeta \gtrsim 3.7$, the correlation length between spinons (which is in principle unphysical, as spinons are now confined) becomes dominant. ${ }^{7}$ Finally, let us point out that while on the one hand, the critical point $\zeta_{c} \approx 2.28 \mathrm{did}$ not change significantly as compared to the trimer model (where $\zeta_{c} \approx 2.19$ ), ${ }^{8}$ the implications on how the inequivalent ways to resolve folded trimers affect the phase is rather different. Since at the SU(3) point, all folded trimers are replaced by the same $\mathrm{SU}(3)$ singlet, the weight $\zeta$ which appears when resolving a folded trimer as three distinct trimers is $\zeta=3$ rather than $\zeta=\sqrt{3}$, and thus, the corresponding $\mathrm{SU}(3)$ model is in the symmetry broken crystalline rather than the topological phase, as indeed reported in Ref. [19].

Let us now discuss parent Hamiltonians in the context of the SU(3) model. There are several aspects. On the one hand, we are interested in the Hamiltonian for the SU(3) model itself, but on the other hand, we will also discuss how the parent Hamiltonians for the trimer model are affected if we equip the trimers with actual $\mathrm{SU}(3)$ singlets (or some other sufficiently symmetric tripartite entangled state), while keeping different trimer configurations orthogonal. More generally, we will discuss parent Hamiltonians along the entire interpolation family $\mathcal{P}_{1} \rightarrow \mathcal{P}_{2} \rightarrow \mathcal{P}_{3}$, where the points $\mathcal{P}_{3}$ and $\mathcal{P}_{2}\left(\mathcal{P}_{1}\right)$ correspond to the two aforementioned cases.

Why is the Hamiltonian-as discussed in Sec. IIIaffected if we equip the trimers, for which we have been hitherto using the arrow representation, with a tripartite entangled state? To this end, consider the transition between the two configurations in Fig. 4(b), and the corresponding Hamiltonian term. In the arrow representation (left), this is a one-hexagon move, where we just need to change the arrows on the hexagon itself from blue to red. On the other hand, if we equip the trimers with entangled states, we must in addition update the entangled states placed on top of the trimers. This can be done in different ways, such as the one indicated by green arrows [Fig. 4(b), right], but regardless of how it is done, it requires to act on at least one of the outer vertices. This situation occurs precisely if only the inner vertex of a trimer lies on the hexagon, and the $\circ$ on the inner vertex is changed in the transition. In that case, the degrees of freedom on the two outer vertices of the initial trimer, which are entangled in the initial state, belong to two different trimers after the move, and are thus part of two different entangled states; hence, disentangling the degrees of freeom requires to act on

\footnotetext{
${ }^{7}$ Using the Cauchy-Schwarz inequality, one can see that the spinon correlation length should be upper bounded by the spinon confinement length, cf. Ref. [26].

${ }^{8}$ Note that a similar robustness was observed when comparing the effect of vison doping in dimer versus RVB states [30].
}

at least one of them. In all other cases, the trimer pattern can be changed without touching the vertices outside the hexagon, since the outside vertices remain part of the same trimer. ${ }^{9}$ For one-hexagon moves, in the worst case [Fig. 4(c)], this requires to act on eight spins, while the required two-hexagon move can be implemented by acting on $12=11+1$ spins [two hexagons plus the vertex marked by the dashed green circle in Fig. 4(a)]. Note that since the local terms $h_{\bigcirc}$ and $h_{\infty}$ in the Hamiltonian are of the form $h_{\bullet}=\mathbb{1}-\sum\left|\chi_{k}\right\rangle\left\langle\chi_{k}\right|$, with the $\left|\chi_{k}\right\rangle$ the superposition of coupled configurations (and thus 8or 12-local), the total Hamiltonian can indeed be expressed as a sum of 8-local or 12-local terms, respectively.

Let us now return to the Hamiltonian along the interpolation $\mathcal{P}_{1} \rightarrow \mathcal{P}_{2} \rightarrow \mathcal{P}_{3}$. Along the line $\mathcal{P}_{1} \rightarrow \mathcal{P}_{2}$, the model corresponds to the trimer model, where the trimers have been replaced by different entangled states depending on the interpolation parameter. Thus, our discussion above applies to the whole interpolation, and implies the existence of an 8-body and 12-body Hamiltonian acting around one and two hexagons, respectively, depending on whether we are willing to accept an additional isolated "ice" sector on top of the topologically degenerate ground space. ${ }^{10}$ As we move further and interpolate $\mathcal{P}_{2} \rightarrow \mathcal{P}_{3}$, orthogonality of different trimer configurations is lost. However, since the arrow information in $\mathcal{P}_{2}$ is smoothly removed through a "filtering" map $\Lambda=(1-\theta) \mathbb{1}+$ $\theta|+\rangle\langle+|$ on the arrow degree of freedom (with |\rangle$+$ the even weight superposition) [12,19], the PEPS at any point along the interpolation is related to the state $\mathcal{P}_{2}$ by a local invertible map, except for the final point $\mathcal{P}_{3}$ itself. Since the Hamiltonian is frustration free, this implies that we can change the local terms in the Hamiltonian correspondingly (using the inverse map) in a smooth way without changing the structure of the topological ground space which remains ninefold degenerate. However, this only works if we apply this transformation to each of the terms $h_{\bigcirc}, h_{\bigcirc}=\mathbb{1}-\sum\left|\chi_{k}\right\rangle\left\langle\chi_{k}\right|$ as a whole, rather than the terms $\left|\chi_{k}\right\rangle\left\langle\chi_{k}\right|$ individually, and thus yields a 12-local or 19-local Hamiltonian instead, ${ }^{11}$ just as for the $\mathrm{SU}(3)$ model of Ref. [19]. Finally, for the SU(3)-point $\mathcal{P}_{3}$, we can construct a Hamiltonian which yet again consists of 12- or 19-body terms by taking the limit of the parent Hamiltonian along the interpolation; $;{ }^{12}$ however, we cannot

\footnotetext{
${ }^{9}$ Note that we assume that entangled state possesses a symmetry with respect to exchanging the outer vertices of the trimer, cf. footnote 10 .

${ }^{10}$ Note that the state which we put on the trimers (footnote 6) is antisymmetric under exchanging the outer vertices, and thus, a Hamiltonian acting on one/two outer vertices as shown in Fig. 4 is sufficient to update trimer configurations.

${ }^{11}$ Concretely, for a wave function $|\Psi\rangle$ with Hamiltonian $\sum h_{i}$, where $h_{i} \geqslant 0$ and $h_{i}|\Psi\rangle=0$, an invertible deformation $\left|\Psi^{\prime}\right\rangle=$ $\Lambda^{\otimes N}|\Psi\rangle$ on the wave function corresponds to a deformed parent Hamiltonian $h_{i}^{\prime}=\left(\left(\Lambda^{-1}\right)^{\dagger}\right)^{\otimes k} h_{i}\left(\Lambda^{-1}\right)^{\otimes k}$, with the tensor product ${ }^{\otimes k}$ acting on the sites on which $h_{i}$ acts. Note that $h_{i}^{\prime}$ can be replaced by a projector $h_{i}^{\prime \prime}$ with the same kernel while keeping smoothness in the deformation (as eigenspace projectors of analytic maps are analytic as well [33], Thm. 6.1]). See Ref. [12] for a detailed discussion.

${ }^{12}$ The argument is similar to the previous footnote 11 . Since the ground states $\left|\chi_{k}\right\rangle$ are polynomials in the deformation parameter and
} 
rule out that this Hamiltonian exhibits additional ground states. ${ }^{13}$

Finally, we have investigated the behavior of the SU(3) wave function as a function of $\zeta$ as an ansatz for SU(3) Hamiltonians with 3-body interactions on triangles. Any such 3-body term can be decomposed as a sum of four projections $h_{\mathbf{1}}, h_{\mathbf{1 0}}, h_{\mathbf{8}_{L}}$, and $h_{\mathbf{8}_{R}}$ onto the corresponding irreps (here, $\boldsymbol{8}_{\boldsymbol{L}}$ and $\mathbf{8}_{R}$ denote the $\mathbf{8}$ irreps with angular momentum $\pm 2 \pi / 3$, respectively); in particular, the Heisenberg-type Hamiltonian of Ref. [34] (the sum over permutations of nearest neighbors) corresponds to $h_{\text {Heis, } \Delta}=3\left(h_{10}-h_{1}\right)$. The expectation values for the corresponding terms are shown in Fig. 7(b), where energies for up and down triangles are plotted with the corresponding symbol. One can clearly identify the symmetry breaking phase transition, and the fact that in the crystalline phase, the up-triangles hold the folded trimers and are thus in a singlet. ${ }^{14}$ Unfortunately, we found that for all SU(3)-invariant three-body interactions which we considered, either the $\zeta=0$ or the $\zeta \rightarrow \infty$ point provide the lowest variational energy, making it unlikely that the wave function accurately captures the way in which the physics of SU(3) models with three-body interactions across triangles depends on the interactions.

\section{CONCLUSIONS}

In this work, we have introduced and studied quantum trimer models and resonating SU(3)-singlet models on the kagome lattice. We have devised an arrow representation for the trimer model which provides a direct mapping to $\mathbb{Z}_{3}$ loop models. While the loop pattern is missing a configuration, we showed that the full space of $\mathbb{Z}_{3}$-configurations (that is, $\mathbb{Z}_{3}$-injectivity) is recovered under blocking. This allowed us to combine analytical tensor network tools with a microscopic analysis to devise simple parent Hamiltonians with 6-body or 11-body interactions: While the 11-body terms gives rise to the correct ninefold ground space degeneracy, restricting to 6-body terms alone only gives rise to six additional isolated "ice" states with frozen trimers which do not couple to any of the remaining configurations and which we thus expect to be strongly suppressed.

thus analytic also around the final point, it follows that the ground space changes analytically and thus the limit of the projector-valued parent Hamiltonians $h_{i}^{\prime \prime}$ above exists.

${ }^{13}$ Parent Hamiltonians can also be constructed by using that the tensor network on one star (12 sites), seen as a map from virtual to physical system, has constant rank everywhere except at the point $\mathcal{P}_{3}$, and thus, an invertible map on disjoint stars maps it to the orthogonal trimer model, which however yields a Hamiltonian with significantly larger locality [12]. A similar argument, using that the PEPS map on a star is $G$-injective, was used for the SU(3) model in Ref. [19], where it gave a 19-body Hamiltonian; the reason why we can apply a much more direct argument which maps the model to the trimer point and thus yields simpler parent Hamiltonians, while not requiring numerical checks of $G$-injectivity on large patches, lies in the fact that unlike in Ref. [19], no big entangled clusters appear in the superposition (1), since the $000 \equiv \overline{\mathbf{3}} \otimes \overline{\mathbf{3}} \otimes \overline{\mathbf{3}}$ configuration is missing.

${ }^{14}$ The symmetry breaking pattern is controlled by biasing the initial state of the iMPS boundary.
We have subsequently studied the phase diagram of the model by combining analytical arguments with numerical study. We have found that a key role is played by the relative weight $\zeta$ of folded (on-triangle) versus unfolded trimers. For sufficiently large $\zeta$, the system displays a conventional symmetry-broken phase in which all SU(3) singlets are localized on one type of triangles. In the intermediate regime $0<\zeta<\zeta_{c} \approx 2.19$, we identified a topological phase with $\mathbb{Z}_{3}$ topological order. Finally, for $\zeta=0$, we found an additional $\mathrm{U}(1)$ symmetry, indicative of critical behavior which we confirmed numerically.

We have finally equipped the trimer model with SU(3) singlets and shown how these give rise to only slightly enlarged 8- or 12-body Hamiltonians. Using this as a starting point, we devised an interpolation where we erase the trimer (arrow) information, leaving us with a pure resonating $\mathrm{SU}(3)$-singlet model with the fundamental representation per site. We numerically studied this model for $\zeta=1$ along the interpolation from the trimer point, and found that it is a $\mathbb{Z}_{3}$ topological spin liquid in the same phase as the trimer model and the $\mathbb{Z}_{3}$ RG fixed point loop model. Investigating the dependence on $\zeta$ resulted in a phase diagram simlar to the one above, with only a slight change in $\zeta_{c} \approx 2.28$; yet this implies that a model where we were to place $\mathrm{SU}(3)$ singlets in three different ways on any triangle would be trivial rather than topologically ordered, unlike for the quantum trimer model.

Several open questions remain. For one thing, it would be interesting to understand if there is a connection between the geometry of the lattice and the range of phases it can host, similar to the bipartite vs. nonbipartite phenomenon for $\mathrm{SU}(2)$ RVBs. Similarly, the extension of the trimer construction to $\mathrm{SU}(N)$ and $N$-mers holds a wide range of interesting questions, from the nature of their quantum order or the role played by the lattice to the effect of the growing number of inequivalent trimer weights. Finally, our findings suggest that our ansatz, even though it exhibits a symmetry broken phase, does not capture the physics of SU(3) Heisenberg models beyond mean field, leaving the quest for a suitable $\mathrm{SU}(3)$ ansatz open.

\section{ACKNOWLEDGMENTS}

We acknowledge helpful discussion with Hong-Hao Tu. This work has received support from the European Union's Horizon 2020 program through the ERC-StG WASCOSYS (No. 636201), and from the DFG (German Research Foundation) under Germany's Excellence Strategy (EXC2111390814868).

\section{APPENDIX A: TWO-HEXAGON PARENT HAMILTONIAN}

\section{Setting and goal}

In this Appendix, we show that the trimer model appears as the topologically ninefold degenerate ground state of a Hamiltonian

$$
H=\sum h_{\prec}+\sum h_{\infty}
$$

cf. Eq. (3), where $h_{\prec}$ and $h_{\infty}$ act on the degrees of freedom adjacent to a vertex and to a pair of hexagons, respectively. As discussed in the main text, $h_{\prec}$ ensures that the ground 
(a)

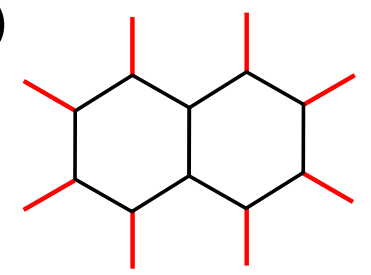

(b)

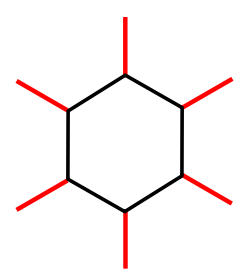

FIG. 8. (a) The two-hexagon parent Hamiltonian acts on the interior indices of the two hexagons (black edges), and couples all interior configuations compatible with the boundary configurations (red edges); note that the latter can be uniquely inferred from the interior configurations. In addition, Hamiltonian terms enforcing the vertex constraints are required. (b) The one-hexagon Hamiltonian only acts on the interior degrees of freedom of one hexagon. It leads to decoupled "ice" sectors [Fig. 4(a)], see Appendix B.

space is spanned by valid $\mathbb{Z}_{3}$ loop configurations, while $h_{\infty}$ couples different loop configurations which only differ on the two hexagons (that is, its ground space consists of properly weighted superpositions of the corresponding configurations), Fig. 8(a).

What remains to be shown is that any two configurations in the same topological sector can be coupled by a sequence of two-hexagon moves $h_{\infty}$. By construction, all the topological sectors are ground states of $\sum h_{\infty}$, and the fact that all configurations are coupled implies that they can only appear in a superposition of all allowed loop patterns with the correct relative weight, that is, there are no other ground states (which would imply decoupled sectors of loop patterns).

As discussed in the main text, a parent Hamiltonian with terms $h^{\prime}$ constructed on a region sufficiently larger than the injective region of Fig. 3(f) has the correct ground space structure $[12,20,28]$. On the other hand, the ground space of this $h^{\prime}$ is precisely spanned by the allowed loop configurations on that region, where configurations which only differ inside that region are coupled. Differently speaking, being able to induce transitions between any two configurations on the region supporting $h^{\prime}$ is sufficient to couple any two configurations in the same sector.

What remains to be shown is that two-hexagon moves allow to construct any desired transition on a larger region. This is what we will do in this Appendix.

\section{Definitions and notation}

For the purpose of the proof, we will consider hexagonshaped blocks as the one shown in Fig. 9, and we will show that using two-hexagon moves, any move on such hexagons (of any given size) can be achieved. In particular, this region can be chosen large enough to contain the aforementioned parent Hamiltonian $h^{\prime}$, which yields all transitions induced by $h^{\prime}$, and thus implies the existence of transitions between all configurations in the same topological sector.

Here, "move" denotes any transition between two loop configurations on the edges inside the respective region, in such a way that both configurations are consistent as parts of a larger system. This is illustrated in Fig. 8(a) for a two-hexagon move, which maps any configuration of the 11 black edges to any other configuration which is consistent with the same

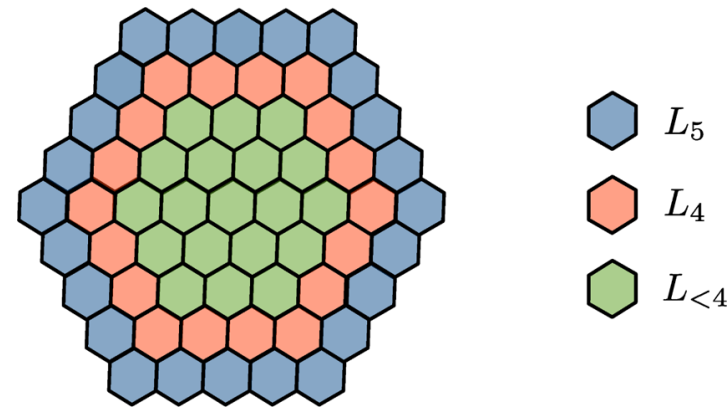

FIG. 9. Geometry considered in the proof of the two-hexagon parent Hamiltonian. The blue and red hexagons form a 5-ring and a 4-ring, respectively; a height configuration assigned to these hexagons is denoted by $L_{5}$ and $L_{4}$. The green hexagons form a 3-disk, with configuration $L_{<4}=\left(L_{3}, L_{2}, L_{1}\right)$.

value of the surrounding red legs. Note that the value of the red legs can be inferred from the black degrees of freedom at the inside, i.e., such a move does not require to access the exterior indices.

It will be convenient to work in the height representation of configurations, that is, with plaquette variables. Let us introduce some language: We will denote a hexagon-shaped ring of $6 n-6$ hexagons, such as the blue and red rings in Fig. 9, as an $n$-ring ( $n$ counts the hexagons at one edge) e.g., the blue hexagons in Fig. 9 forms a 5-ring, and the red hexagons form a 4-ring. The union of all $m$-rings with $m \leqslant n$ will be called an $n$-disk; e.g., the green region is a 3 -disk. In the height representation, each hexagon is assigned a height value, with the constraint that three identical heights adjacent to each other are forbidden, Fig. 3(b). Then, a move acting on a region consists of replacing the height configuration inside that region by another one, keeping the outside configuration fixed, in such a way that no forbidden configuration appears. In particular, a two-hexagon move amounts to changing the height value of two adjacent plaquettes, and a single-hexagon move that of a single hexagon. Note that we only consider concentric rings as in Fig. 9, making the notion of $n$-rings and $n$-disks unique.

In the following, we will show inducively that if two configurations on an $n$-disk agree on the exterior $n$-ring, we can make them agree on their interior $(n-1)$-disks by a sequence of local moves acting on two adjacent hexagons each. Since the region on which the original parent Hamiltonian $h^{\prime}$ acts is contained in an $n$-disk for sufficiently large $n$ (and thus couples at most all configurations on that $n$-disk), this implies that a two-hexagon parent Hamiltonian is sufficient to couple any two valid height configurations.

Let us introduce a notation for height configurations on the concentric $n$-rings and $n$-disks. First, we denote the height configuration on the $n$-ring by $L_{n}$. We say that two configurations $L_{n}$ and $L_{n-1}$ are consistent if no forbidden configurations [three equal adjacent heights, Fig. 3(b)] appear. For two consistent configurations $L_{n}$ and $L_{n-1}$, we denote the joint configuration on the $n$-ring and the $(n-1)$-ring by $\left(L_{n}, L_{n-1}\right)$, and so forth. Finally, $L_{<n}=\left(L_{n-1}, L_{n-2}, \ldots, L_{1}\right)$ denotes the joint configuration of the $(n-1)$-disk; and thus, e.g., $\left(L_{n+1}, L_{<n+1}\right)$ or $\left(L_{n+1}, L_{n}, L_{<n}\right)$ the joint configuration on the $(n+1)$-disk. 
Whenever we use this notation, this implies consistency of the configuration. For example, a configuration in Fig. 9 would be denoted by $\left(L_{n+1}, L_{n}, L_{<n}\right)$ with $n=4$, where $L_{n+1}$ is the configuration on the blue, $L_{n}$ the configuration on the red, and $L_{<n}$ the configuration on the green hexagons.

When two configurations $\left(L_{n}, L_{<n}\right)$ and $\left(L_{n}, L_{<n}^{\prime}\right)$ are related by local moves on the $(n-1)$-disk we write $\left(L_{n}, L_{<n}\right) \longleftrightarrow\left(L_{n}, L_{<n}^{\prime}\right)$. Here, local moves are those which are generated by two-hexagon moves on the $(n-1)$-disk. That is, either elementary two-hexagon moves, or moves for which we have already shown that they can be constructed from two-hexagon moves. Clearly, being related by local moves is transitive.

\section{Overview of the proof}

We will prove the following.

Theorem 1. For any $n \geqslant 3, L_{n}$, and $L_{<n}, L_{<n}^{\prime}$ consistent with $L_{n}$, the transition from $L_{<n}$ to $L_{<n}^{\prime}$ can be realized through local (two-hexagon) moves:

$$
\forall L_{n}, L_{<n}, L_{<n}^{\prime}:\left(L_{n}, L_{<n}\right) \longleftrightarrow\left(L_{n}, L_{<n}^{\prime}\right)
$$

The moves only act on the inside, i.e., the $(n-1)$-disk, and leave $L_{n}$ unchanged throughout.

Proof. The proof will proceed by induction, with induction hypothesis (A2). That (A2) holds for $n=3$ can be checked by brute force. Notably, this is the only step where twohexagon moves are required; we will get back to this point in Appendix B.

Let us now prove the induction step, that is, if (A2) holds for $n \geqslant 3$, it also holds for $n^{\prime}=n+1$. To this end, given configurations $\left(L_{n+1}, L_{n}, L_{<n}\right)$ and $\left(L_{n+1}, L_{n}^{\prime}, L_{<n}^{\prime}\right)$ which we want to connect through local moves, we first construct a sequence $L_{n}=L_{n}^{(0)}, L_{n}^{(1)}, \ldots, L_{n}^{(N)}=L_{n}^{\prime}$ with the following properties:

(i) $L_{n}^{(i)}$ and $L_{n}^{(i+1)}$ differ only on a single hexagon.

(ii) For all $i,\left(L_{n+1}, L_{n}^{(i)}\right)$ is consistent.

The construction of this sequence is given in Appendix A 4. Next, for $i=1, \ldots, N-1$, we show in Appendix A 5 that since $L_{n}^{(i)}$ and $L_{n}^{(i+1)}$ only differ on a single hexagon, we can construct an $L_{<n}^{(i)}$ such that

(iii) Both $\left(L_{n}^{(i)}, L_{<n}^{(i)}\right)$ and $\left(L_{n}^{(i+1)}, L_{<n}^{(i)}\right)$ are consistent.

Then, we have that

$$
\begin{aligned}
& \left(L_{n+1}, L_{n}, L_{<n}\right) \stackrel{*}{\longleftrightarrow}\left(L_{n+1}, L_{n}^{(0)}, L_{<n}^{(0)}\right) \\
& \stackrel{\bigcirc}{\longleftrightarrow}\left(L_{n+1}, L_{n}^{(1)}, L_{<n}^{(0)}\right) \\
& \stackrel{*}{\longleftrightarrow}\left(L_{n+1}, L_{n}^{(1)}, L_{<n}^{(1)}\right) \\
& \stackrel{\ominus}{\longleftrightarrow}\left(L_{n+1}, L_{n}^{(2)}, L_{<n}^{(1)}\right) \\
& \longleftrightarrow \quad \ldots . \\
& \stackrel{\bigcirc}{\longleftrightarrow}\left(L_{n+1}, L_{n}^{(N)}, L_{<n}^{(N-1)}\right) \\
& \stackrel{*}{\longleftrightarrow}\left(L_{n+1}, L_{n}^{\prime}, L_{<n}^{\prime}\right) \text {. }
\end{aligned}
$$

Here, we have used the induction hypothesis (A2) in the step marked with a star, and a single-hexagon move on the one hexagon on which $L_{n}^{(i)}$ and $L_{n}^{(i+1)}$ differ [condition (i)] in the
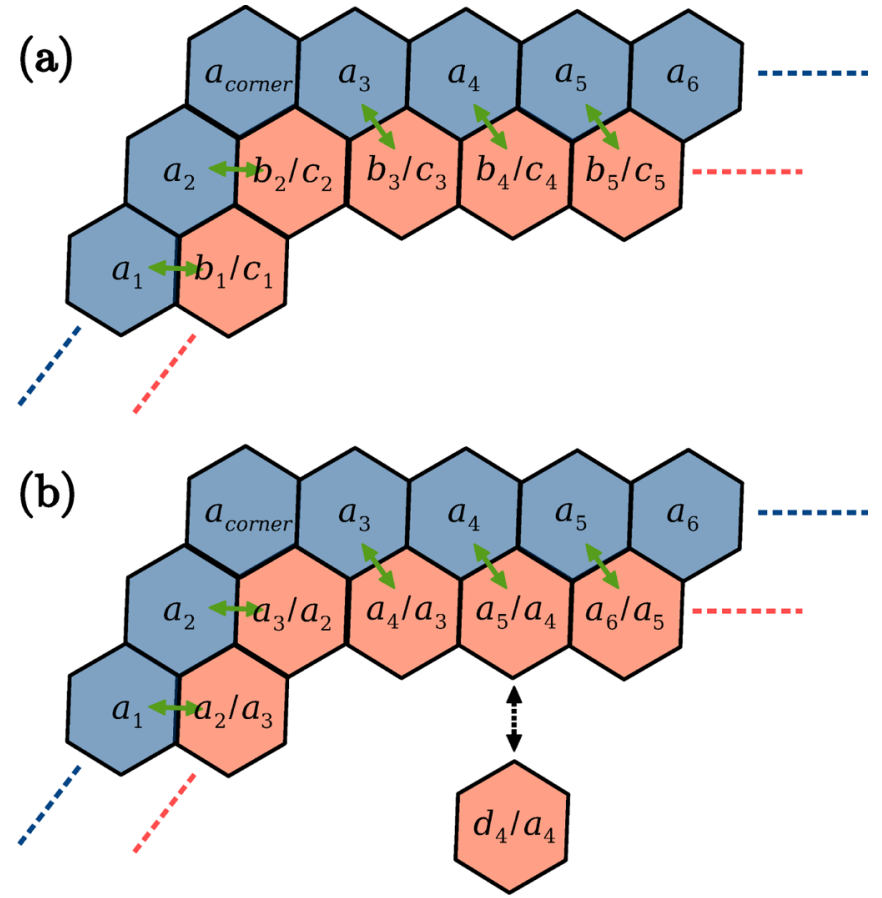

FIG. 10. (a) Setting considered when constructing the sequence $L_{n}^{(k)}$, see Appendix A 4. The configuration $L_{n+1}$ of the $(n+1)$-ring (blue) is denoted by $a_{i}$, and those of the two configurations $L_{n}$ and $L_{n}^{\prime}$ of the $n$-ring (red) which we want to connect by $b_{i}$ and $c_{i}$, respectively. Green arrows indicate how the plaquette labels $i$ are assigned relatively between the two rings. (b) A setting $L_{n}, L_{n}^{\prime}$ where all plaquettes are unflippable [where $b_{i-1}=c_{i}=a_{i}$ for all $i$, condition (A3b)]. To resolve this situation, we insert an additional configuration where we change one plaquette in the initial state as indicated, with $d_{4} \neq a_{4}, a_{5}$. The new configuration now has flippable plaquettes.

steps marked with a hexagon. Note that conditions (ii) and (iii) imply that all intermediate configurations are consistent.

This shows that if (A2) holds for $n$, it also holds for $n^{\prime}=$ $n+1$, and thus completes the proof.

\section{Construction of $L_{n}^{(0)}, \ldots, L_{n}^{(N)}$}

Here, we show the following: Given configurations $L_{n+1}$, $L_{n}$, and $L_{n}^{\prime}$, such that $L_{n+1}$ is consistent with both $L_{n}$ and $L_{n}^{\prime}$, we construct a sequence $L_{n}=L_{n}^{(0)}, L_{n}^{(1)}, \ldots, L_{n}^{(N)}=L_{n}^{\prime}$ such that all $L_{n}^{(k)}$ are consistent with $L_{n+1}$, and consecutive $L_{n}^{(k)}$ only differ on a single hexagon.

Our construction will proceed sequentially, starting with $k=0$, and $L_{n}^{(0)}=L_{n}$. In each step-labeled by $k$-we compare $L_{n}^{(k)}$ with $L_{n}^{\prime}$. We denote the height values of $L_{n+1}$ by $a_{i}$ and the height values of $L_{n}^{(k)}$ and $L_{n}^{\prime}$ by $b_{i}$ and $c_{i}$, respectively, as indicated in Fig. 10(a). Here, the plaquette labels $i$ are chosen such that the label of any hexagon in the $n$-ring equals that of the hexagon in the $(n+1)$-ring to its left, as seen from the center [cf. the green arrows in Fig. 10(a)]. The height values of the corners of the $(n+1)$-ring will not be required. We now proceed by identifying a flippable plaquette $i$, that is, a plaquette for which $b_{i} \neq c_{i}$ and where we can change $b_{i}$ to $c_{i}$ in a way where the resulting configuration is consistent, and then define $L_{n}^{(k+1)}$ to be the resulting configuration on the 
$n$-ring (that is, $L_{n}^{(k+1)}$ equals $L_{n}^{(k)}$ and thus $b_{j}$ on all plaquettes $j$ except for $i$, which is set to $c_{i}$ ).

Clearly, this protocol will succeed in transforming $L_{n}^{(0)}$ to $L_{n}^{\prime}$ if we can make sure that at every step $k$, there is at least one flippable plaquette, since at most all the $6 n-6$ plaquettes on the $n$-ring need to be flipped. Let us thus consider under which condition a plaquette is unflippable. This can happen in two ways: Either, we already have

$$
b_{i}=c_{i},
$$

or after changing $b_{i}$ to $c_{i}$, the configuration becomes inconsistent across one of the two vertices,

$$
\begin{gathered}
b_{i-1}=a_{i}=c_{i} \quad \text { or } \\
c_{i}=a_{i+1}=b_{i+1} .
\end{gathered}
$$

If (and only if) either of the equations (A3) is satisfied, then the plaquette $i$ is unflippable.

Let us now analyze the situation where all hexagons are unflippable. First, if $b_{i}=c_{i}$ for all hexagons, then $L_{n}^{(k)}=L_{n}^{\prime}$ and we are done. Thus, we can pick an $i$ for which $b_{i} \neq c_{i}$, i.e. (A3a) fails. Hence, either (A3b) or (A3c) has to hold. Assume w.l.o.g. that (A3b) holds. Since $\left(L_{n+1}, L_{n}^{\prime}\right)$ is consistent, $\left\{c_{i-1}, a_{i}, c_{i}\right\}$ cannot all be equal, which together with (A3b) implies $b_{i-1}=a_{i}=c_{i} \neq c_{i-1}$. Specifically, this means that

$$
b_{i-1} \neq c_{i-1} \quad \text { and } \quad c_{i-1} \neq a_{i},
$$

and thus, neither (A3a) nor (A3c) can hold for the hexagon $i-1$, such that (A3b) must hold for the hexagon $i-1$ as well. By continuing this way, we find that if all hexagons are unflippable and not all $b_{i}=c_{i}$, then the following two properties hold:

(a) either (A3b) holds for all $i$, or (A3c) holds for all $i$;

(b) for all $i, b_{i} \neq c_{i}$.

The case where (A3b) holds for all $i$ is illustrated in Fig. 10(b). Note that, e.g., the validity of (A3b) for all $i$ implies that (A3c) cannot hold anywhere, since otherwise forbidden configurations would appear.

One important point is that point (b) above tells us that the situation where all hexagons are unflippable can only appear in the very beginning, $k=0$. After the first step, $k \geqslant 1$, some of the hexagons in $L_{n}^{(k)}$ have already been flipped and therefore equal those in $L_{n}^{\prime}, b_{i}=c_{i}$.

So what if when considering $L_{n} \equiv L_{n}^{(0)}$ and $L_{n}^{\prime}$, all plaquettes are unflippable, and thus point (a) above is satisfied? We will deal with that by creating an additional configuration $L_{n}^{(1)}$ (obtained by flipping one plaquette in $L_{n}^{(0)}$ ) such that comparing $L_{n}^{(1)}$ and $L_{n}^{\prime}$, there is a flippable hexagon. W.l.o.g., we will assume that (A3b) holds for all $i$, Fig. 10(b); note that this implies that (A3c) holds nowhere. Then, we pick any hexagon $i$ on the $n$-ring and define $L_{n}^{(1)}$ to be equal to $L_{n}^{(0)}$ everywhere except on hexagon $i$, to which we assign the value $d_{i} \neq a_{i}, a_{i+1} .\left(L_{n+1}, L_{n}^{(1)}\right)$ is consistent, as can be seen from Fig. 10(b), where $i=4$, i.e., the $a_{5}$ in the marked hexagon is now replaced by $d_{4}$ as indicated. By construction, $L_{n}^{(1)}$ differs from $L_{n}^{(0)}$ on a single site. Finally, when comparing $L_{n}^{(1)}$ and $L^{\prime}$, the plaquette $i+1$ is flippable, since condition (A3b) no
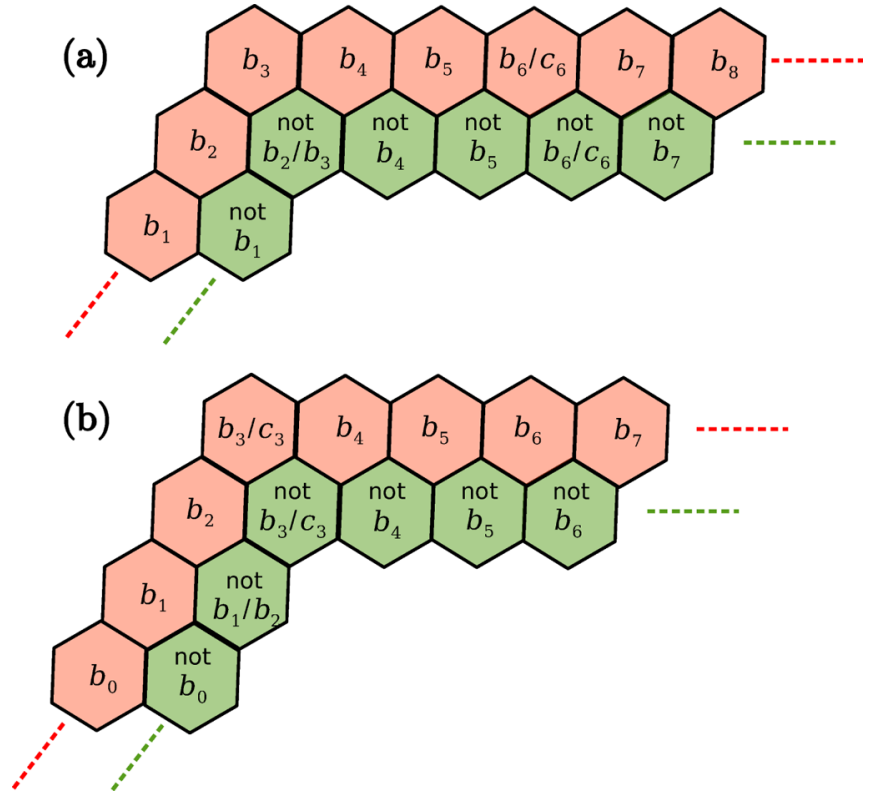

FIG. 11. Construction of $L_{n-1}^{(i)}$ (green hexagons) in the case where $L_{n}^{(i)}$ and $L_{n}^{(i+1)}$ (red hexagons) differ (a) at an edge or (b) at a corner of the $n$-ring, see Appendix A 5.

longer holds by choice of $d_{i}$, (A3c) did not hold to start with, and $b_{i} \neq c_{i}$.

\section{Construction of the $L_{n-1}^{(i)}$}

In this Appendix, we show that, given $L_{n} \equiv L_{n}^{(i)}$ and $L_{n}^{\prime} \equiv$ $L_{n}^{(i+1)}$ which differ on one hexagon, $n \geqslant 3$, we can construct $L_{<n} \equiv L_{<n}^{(i)}$ such that both $\left(L_{n}, L_{<n}\right)$ and $\left(L_{n}^{\prime}, L_{<n}\right)$ are consistent.

First, consider the case where $n \geqslant 4$. We set out by constructing $L_{n-1}$ such that $\left(L_{n}, L_{n-1}\right)$ and $\left(L_{n}^{\prime}, L_{n-1}\right)$ are consistent. There are two possibilities. First, the hexagon on which $L_{n}$ and $L_{n}^{\prime}$ differ is not at a corner of the $n$-ring, such as shown in Fig. 11(a) for the outer (red) hexagon with values $b_{6} / c_{6}$ (the values on which $L_{n}$ and $L_{n}^{\prime}$ agree are denoted by $b_{i}$ ). Then, construct $L_{n-1}$ as shown by the inner (green) hexagons in Fig. 11(a), where "not $x$ " means that we can choose any value except $x$, and "not $x / y$ " any value except $x$ and $y$. By this choice, no three hexagons around a vertex can have the same value for either $L_{n}$ or $L_{n}^{\prime}$. In the second case, where the hexagon which differs is at a corner of the $n$-ring, we construct $L_{n-1}$ as shown in Fig. 11(b). While now it is possible that the hexagon at the corner of the $(n-1)$-ring has the value $b_{2}$, the choice of the hexagon below as not $b_{1} / b_{2}$ (rather than just not $b_{1}$ ) ensures that also across that vertex, the three hexagons cannot all have the same value.

Now that we have constructed $L_{n-1}$, we can use the same prescription to construct $L_{n-2}$ such that $\left(L_{n-1}, L_{n-2}\right)$ is consistent (ignoring the $L^{\prime}$-part in the construction), and so forth. Once we have arrived at $L_{3}$, we can use the argument from the $G$-injectivity proof in the main text, Fig. 3(e), to construct a consistent $L_{<3}$. Putting all the layers $\left(L_{n-1}, L_{n-2}, \ldots, L_{3}, L_{<3}\right)$ together, we obtain $L_{<n}$ such that both $\left(L_{n}, L_{<n}\right)$ and $\left(L_{n}^{\prime}, L_{<n}\right)$ are consistent. 


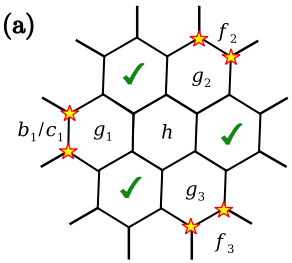

(b)

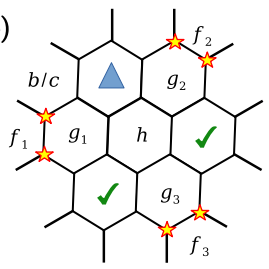

(c)

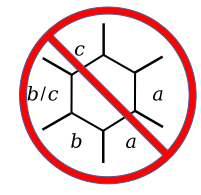

FIG. 12. Construction of an interior configuration $L_{<3}$ consistent with two outer configurations $L_{3}$ and $L_{3}^{\prime}$ differing on one plaquette, see Appendix A 5. (a) Situation where the differing plaquette is at the corner. (b) Situation where the differing plaquette is on the edge. (c) Forbidden configuration (plus rotations/reflections) when the plaquette on the left can take two values; here, $a, b, c$ are all different.

The above argument fails for $n=3$ where we are given $L_{3}$ and $L_{3}^{\prime}$ and want to construct an $L_{2}$ consistent with both, since the 2-ring consists exclusively of corners. To cover the $n=3$ case, we will instead provide a direct construction of $L_{<3}$, in close analogy to the argument used in the main text to construct a consistent interior of an arbitrary 3-ring [Fig. 3(e)].

Yet again, we need to consider two cases. The first is where $L_{3}$ and $L_{3}^{\prime}$ differ at the corner of the 3-ring, see Fig. 12(a). We want to construct a configuration on the interior that is consistent with both $b_{1}$ and $c_{1}$. To this end, set $g_{1}=$ $\operatorname{not}\left(b_{1}, c_{1}\right), h=\operatorname{not}\left(g_{1}\right), g_{2}=\operatorname{not}\left(f_{2}, h\right)$, and $g_{3}=\operatorname{not}\left(f_{3}, h\right)$. Then (i) the vertices marked by stars cannot be in the forbidden configuration Fig. 3(b), i.e., three identical adjacent values, and (ii) the hexagons marked with a checkmark cannot be in the forbidden configurations Fig. 3(d), and thus can be assigned a consistent value.

If instead $L_{3}$ and $L_{3}^{\prime}$ differ in the center of an edge, shown in Fig. 12(b), we need to give additional consideration to the value of the hexagon marked with a triangle: unlike for the hexagons marked by a checkmark before, we now need to generalize Fig. 3(d) such as to rule out configurations, which cannot be completed in a way consistent with both $b$ and $c$ at the given plaquette. Those configurations are of the form shown in Fig. 12(c), with $a, b, c$ all different, or variants thereof (reflection and/or exchanging $b$ and $c$ ). Now, in Fig. 12(b), choose $g_{2}=\operatorname{not}\left(f_{2}, \operatorname{not}(b, c)\right)$. This rules out the configuration in Fig. 12(c) around the hexagon marked by the triangle, since there the hexagon opposite of $b / c$ has the value $a=\operatorname{not}(b, c)$. Further, choose $h=\operatorname{not}\left(g_{2}\right), g_{1}=$ $\operatorname{not}\left(f_{1}, h\right)$, and $g_{3}=\operatorname{not}\left(f_{3}, h\right)$. Then, (i) the vertices marked by stars cannot be in the forbidden configuration Fig. 3(b), and (ii) we can fill the hexagons marked with the triangle and the checkmarks in a consistent way, as we have avoided the configurations Figs. 12(c) and 3(d), respectively. This completes the proof.

\section{APPENDIX B: SINGLE HEXAGON HAMILTONIAN}

In this Appendix, we consider parent Hamiltonians which only consist of one-hexagon moves, cf. Fig. 8(b) (together with terms ensuring the $\mathbb{Z}_{3}$ vertex constraints) and show that, with the exception of the six symmetry-related crystalline configurations in Fig. 13 [see also Fig. 4(a)], all other configurations are coupled by one-hexagon moves. (a)

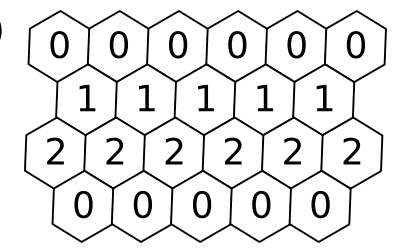

(b)

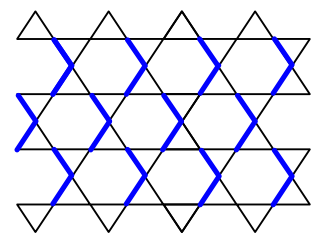

FIG. 13. Crystalline configuration (a) in the height representation and (b) in the trimer representation. These configurations cannot be changed by one-hexagon moves alone.

To start with, it is straightforward to see that the stripe height configuration in Fig. 13(a), corresponding to the crystalline trimer pattern in Fig. 13(b), cannot be changed by one-hexagon moves [see Fig. 4(a) for a two-hexagon move melting such a cluster] and is thus decoupled from any other configuration; we will refer to the corresponding height configuration as "crystalline" as well. There are altogether 6 such configuration related by the lattice rotation symmetry. However, as we will see in the following, these are the only such configurations: All other configurations (within the same sector) can be transformed into each other by one-hexagon moves. First, recall from Appendix A that the only step where we required two-hexagon moves was the start of the induction, that is, Eq. (A2) for $n=3$ :

$$
\left(L_{3}, L_{<3}\right) \longleftrightarrow\left(L_{3}, L_{<3}^{\prime}\right) \text { for all } L_{3}, L_{<3}, L_{<3}^{\prime} .
$$

In the following, we will show that we can always implement such a move anywhere in the system as long as somewhere there is a place where the system is not in the crystalline configuration. In particular, this implies that in order to only keep the nine topological ground states, it is sufficient if we include a single two-hexagon term (which can locally melt the crystal) somewhere in the system.

We are thus interested in the situation where we cannot perform a transformation $\left(L_{3}, L_{<3}\right) \stackrel{Ð . .0}{\longleftrightarrow}\left(L_{3}, L_{<3}^{\prime}\right)$ by a sequence of single-hexagon moves (denoted $\stackrel{\ominus .0}{\longleftrightarrow}$ ) on the inner 2-disk (i.e., $L_{<3}$ vs. $L_{<3}^{\prime}$ ) alone. An exhaustive search shows that for any given $L_{3}$, there are exactly two possibilities: Either all interior configurations $L_{<3}, L_{<3}^{\prime}$ are connected, $\left(L_{3}, L_{<3}\right) \stackrel{\bigcirc .0 \bigcirc}{\longleftrightarrow}\left(L_{3}, L_{<3}^{\prime}\right)$, or there is a single configuration $\hat{L}_{<3}$ which is cannot be connected to any other configuration $L_{<3}$, while all other configurations $L_{<3}, L_{<3}^{\prime} \neq \hat{L}_{<3}$ are still connected by one-hexagon moves, $\left(L_{3}, L_{<3}\right) \stackrel{\ominus \text {.. }}{\longleftrightarrow}\left(L_{3}, L_{<3}^{\prime}\right)$. Moreover, such an $\hat{L}_{<3}$ is necessarily of the form shown in Fig. 14 a (up to symmetry). We will call such configurationwhich are the only ones which require two-hexagon moves to change them-frozen configurations. Note that being frozen is a joint property of $\left(L_{3}, \hat{L}_{<3}\right)$ (as the allowed moves on the interior 2-disk depend on $\left.L_{3}\right)$.

Clearly, if all 2-disks are frozen, the system must be in on of the crystalline states, Fig. 13(a). Thus let us consider the scenario where at least one 2-disk is in a nonfrozen configuration. We will now show that this allows us to "melt" frozen configurations anywhere in the system with only onehexagon moves.

To start with, consider the situation in Fig. 15(a), where the left (red) 2-disk is frozen, and the right (green) 2-disk 

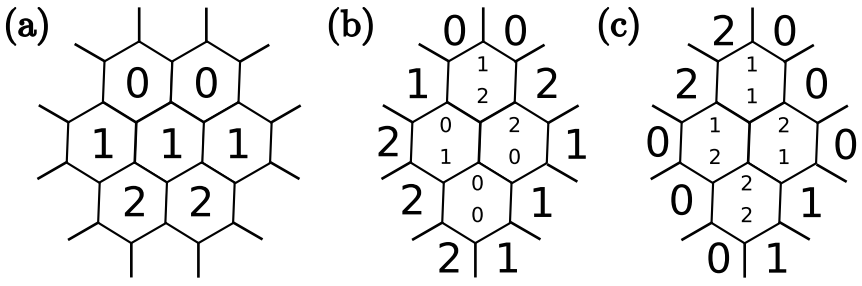

FIG. 14. (a) "Frozen" configurations which cannot be changed by one-hexagon moves on the inner 2-disk for certain boundary conditions. [(b) and (c)] The boundary configurations (up to symmetry) at the intersection of two 2-disks (Fig. 15) which only allow for two interior assignments (top/bottom); all other boundaries have at least three interior assignments.

is not. Our goal is to change the intersection of the two disks (blue) to a different configuration: as there is only a single frozen configuration for a given boundary, this would automatically "melt" the left 2-disk and allow us to subsequently transform it to any desired configuration (except the original frozen one) by one-hexagon moves alone. Since the right 2-disk is not frozen, changing the intersection region is possible by one-hexagon moves on the right 2-disk, unless the desired configuration on the intersection (blue) would amount to the frozen configuration of the right 2-disk. However, an exhaustive search reveals that-given a fixed boundary condition (gray) to the blue intersection-the only cases (up to symmetry) where there are only two choices for the blue intersection are those in Figs. 15(b) and 15(c), and neither of them is is consistent with both a frozen left and right 2-disk in the two configurations. For all other boundary conditions, there are at least three choices for the blue intersection, so there must be one for which neither of the two 2-disks is frozen (as the frozen configurations $\hat{L}_{<3}$ are unique). We can now use one-hexagon moves on the right 2-disk to transform the blue intersection into such a configuration (restoring the plaquettes outside the intersection to their original state); then, the left 2-disk is no longer in the frozen configuration and can be transformed to any other configuration by one-hexagon moves.

In case we want to change a frozen 2-disk with no nonfrozen 2-disk adjacent to it, we choose a path of overlapping (a)

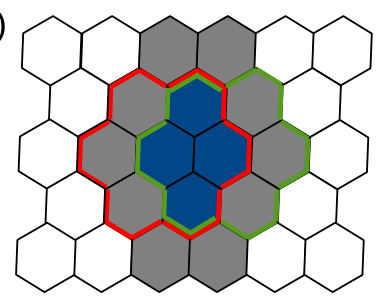

(b)

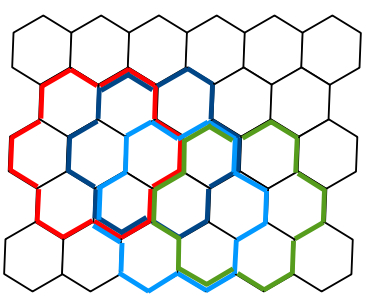

FIG. 15. Setup for "melting" frozen 2-disks by one-hexagon moves (see text). (a) The frozen left (red) 2-disk can be melted if the adjacent right (green) 2-disk is not frozen, by updating the intersection (blue) through one-hexagon moves on the right 2-disk. (b) If the frozen 2-disk (left, red) is inside a frozen cluster, it is connected through a path of frozen 2-disks (blue) to a nonfrozen 2-disk (right, green), and one applies (a) iteratively.

frozen 2-disks connecting it to a nonfrozen 2-disk somewhere in the system; such a path is illustrated in Fig. 15(b). We can then start from the nonfrozen 2-disk and melt the adjacent 2-disk as described above, and continue our way though the chain of 2-disks until we arrive at the original 2-disk which we want to melt. Importantly, while moving through the chain of 2-disks, we can always leave the system in its original state as we move on, as we explain using the diagram in Fig. 15(a). (i) In the first step of the procedure, we are precisely in the situation as before, where the right 2-disk is not frozen and the part outside the intersection is restored to its original state. (ii) In the further steps, however, we start in a situation where initially the left 2-disk is frozen while the right 2-disk is not any more, and at the end of the step, we want to leave the outside part of the right 2-disk in the residual part of the original frozen configuration (though whether it is actually frozen might depend on the environment). We can now use the same argument as before to see that there is at least one configuration on the intersection which is consistent with neither the left nor the right 2-disk being frozen; we can thus use moves on the right 2-disk to transform the outside part to the configuration it had in the original (frozen) configuration, and the intersection to the corresponding third configuration. We thus see that we can use this scheme to melt any frozen 2disk inside a frozen cluster by acting along a one-dimensional path which connects it with the outside of the cluster, while restoring all plaquettes outside the melted 2-disk to their original state.

[1] L. Balents, Spin liquids in frustrated magnets, Nature (London) 464, 199 (2010).

[2] L. Savary and L. Balents, Quantum spin liquids, Rep. Prog. Phys. 80, 016502 (2017).

[3] J. Knolle and R. Moessner, A field guide to spin liquids, Annu. Rev. Condens. Matter Phys. 10, 451 (2019).

[4] P. W. Anderson, Resonating valence bonds: A new kind of insulator?, Mater. Res. Bull. 8, 153 (1973).

[5] R. Moessner and K. S. Raman, Quantum Dimer Models, in Introduction to Frustrated Magnetism, edited by C. Lacroix, P. Mendels, F. Mila, Springer Series in Solid-State Sciences, Vol. 164 (Springer, Berlin, Heidelberg, 2011).
[6] E. Fradkin, Field Theories of Condensed Matter Physics, Field Theories of Condensed Matter Physics (Cambridge University Press, 2013).

[7] R. Moessner and S. L. Sondhi, An RVB Phase in the Triangular Lattice Quantum Dimer Model, Phys. Rev. Lett. 86, 1881 (2001).

[8] G. Misguich, D. Serban, and V. Pasquier, Quantum Dimer Model on the Kagome Lattice: Solvable Dimer-Liquid and Ising Gauge Theory, Phys. Rev. Lett. 89, 137202 (2002).

[9] V. Elser and C. Zeng, Kagome spin-1/2 antiferromagnets in the hyperbolic plane, Phys. Rev. B 48, 13647 (1993). 
[10] A. Kitaev, Fault-tolerant quantum computation by anyons, Ann. Phys. 303, 2 (2003).

[11] M. R. Norman, Colloquium: Herbertsmithite and the search for the quantum spin liquid, Rev. Mod. Phys. 88, 041002 (2016).

[12] N. Schuch, D. Poilblanc, J. I. Cirac, and D. Pérez-García, Resonating valence bond states in the PEPS formalism, Phys. Rev. B 86, 115108 (2012).

[13] Z. Zhou, J. Wildeboer, and A. Seidel, Ground state uniqueness of the twelve site RVB spin-liquid parent Hamiltonian on the kagome lattice, Phys. Rev. B 89, 035123 (2014).

[14] A. V. Gorshkov, M. Hermele, V. Gurarie, C. Xu, P. S. Julienne, J. Ye, P. Zoller, E. Demler, M. D. Lukin, and A. M. Rey, Twoorbital SU(N) magnetism with ultracold alkaline-earth atoms, Nat. Phys. 6, 289 (2010).

[15] F. Scazza, C. Hofrichter, M. Höfer, P. C. D. Groot, I. Bloch, and S. Fölling, Observation of two-orbital spin-exchange interactions with ultracold SU(N)-symmetric fermions, Nat. Phys. 10, 779 (2014).

[16] X. Zhang, M. Bishof, S. L. Bromley, C. V. Kraus, M. S. Safronova, P. Zoller, A. M. Rey, and J. Ye, Spectroscopic observation of SU(N)-symmetric interactions in Sr orbital magnetism, Science 345, 1467 (2014).

[17] H. Lee, Y.-t. Oh, J. H. Han, and H. Katsura, Resonating valence bond states with trimer motifs, Phys. Rev. B 95, 060413(R) (2017).

[18] X.-Y. Dong, J.-Y. Chen, and H.-H. Tu, SU(3) trimer resonatingvalence-bond state on the square lattice, Phys. Rev. B 98, 205117 (2018).

[19] I. Kurecic, L. Vanderstraeten, and N. Schuch, A gapped SU(3) spin liquid with Z3 topological order, Phys. Rev. B 99, 045116 (2019).

[20] N. Schuch, I. Cirac, and D. Pérez-García, PEPS as ground states: Degeneracy and topology, Ann. Phys. 325, 2153 (2010).

[21] F. Verstraete and J. I. Cirac, Valence bond solids for quantum computation, Phys. Rev. A 70, 060302(R) (2004).

[22] F. Verstraete and J. I. Cirac, Renormalization algorithms for Quantum-Many Body Systems in two and higher dimensions, arXiv:cond-mat/0407066.
[23] J. C. Bridgeman and C. T. Chubb, Hand-waving and interpretive dance: An introductory course on tensor networks, J. Phys. A: Math. Theor. 50, 223001 (2017).

[24] D. Perez-Garcia, F. Verstraete, J. I. Cirac, and M. M. Wolf, PEPS as unique ground states of local Hamiltonians, Quantum Inf. Comput. 8, 0650 (2008).

[25] J. Haegeman, V. Zauner, N. Schuch, and F. Verstraete, Shadows of anyons and the entanglement structure of topological phases, Nat. Commun. 6, 8284 (2015).

[26] K. Duivenvoorden, M. Iqbal, J. Haegeman, F. Verstraete, and N. Schuch, Entanglement phases as holographic duals of anyon condensates, Phys. Rev. B 95, 235119 (2017).

[27] N. Schuch, D. Poilblanc, J. I. Cirac, and D. PerezGarcia, Topological Order in PEPS: Transfer Operator and Boundary Hamiltonians, Phys. Rev. Lett. 111, 090501 (2013).

[28] A. Molnar, J. Garre-Rubio, D. Pérez-García, N. Schuch, and J. I. Cirac, Normal projected entangled pair states generating the same state, New J. Phys. 20, 113017 (2018).

[29] M. Iqbal, K. Duivenvoorden, and N. Schuch, Study of anyon condensation and topological phase transitions from a Z4 topological phase using the projected entangled pair states approach, Phys. Rev. B 97, 195124 (2018).

[30] M. Iqbal, H. Casademunt, and N. Schuch, Topological spin liquids: Robustness under perturbations, Phys. Rev. B 101, 115101 (2020).

[31] M. Iqbal, D. Poilblanc, and N. Schuch, Gapped $Z_{2}$ spin liquid in the breathing kagome Heisenberg antiferromagnet, Phys. Rev. B 101, 155141 (2020).

[32] M. M. Rams, P. Czarnik, and L. Cincio, Precise Extrapolation of the Correlation Function Asymptotics in Uniform Tensor Network States with Application to the Bose-Hubbard and XXZ Models, Phys. Rev. X 8, 041033 (2018).

[33] T. Kato, Perturbation Theory for Linear Operators (Springer Berlin Heidelberg, 1966).

[34] P. Corboz, K. Penc, F. Mila, and A. M. Läeuchli, Simplex solids in SU(N) Heisenberg models on the kagome and checkerboard lattices, Phys. Rev. B 86, 041106(R) (2012). 